\title{
Systematic construction of basis invariants in the 2HDM
}

\author{
Andreas Trautner \\ Max-Planck-Institut für Kernphysik, \\ Saupfercheckweg 1, 69117 Heidelberg, Germany \\ Bethe Center for Theoretical Physics und Physikalisches Institut der Universität Bonn, \\ Nussallee 12, 53115 Bonn, Germany \\ E-mail: trautner@mpi-hd.mpg.de
}

ABSTRACT: A new systematic method for the explicit construction of (basis-)invariants is introduced and employed to construct the full ring of basis invariants of the Two-HiggsDoublet-Model (2HDM) scalar sector. Co- and invariant quantities are obtained by the use of hermitian projection operators. These projection operators are constructed from Young tableaux via birdtrack diagrams and they are used in two steps. First, to extract basiscovariant quantities, and second, to combine the covariants in order to obtain the actual basis invariants. The Hilbert series and Plethystic logarithm are used to find the number and structure of the complete set of generating invariants as well as their interrelations (syzygies). Having full control over the complete ring of (CP-even and CP-odd) basis invariants, we give a new and simple proof of the necessary and sufficient conditions for explicit CP conservation in the $2 \mathrm{HDM}$, confirming earlier results by Gunion and Haber. The method generalizes to other models, with the only foreseeable limitation being computing power.

Keywords: Beyond Standard Model, CP violation, Global Symmetries, Renormalization Group

ARXIV EPRINT: 1812.02614 


\section{Contents}

1 Introduction 1

2 Synopsis of jargon $\quad 4$

3 Construction of the building blocks 5

$\begin{array}{lll}4 & \text { CP properties of the building blocks } & 10\end{array}$

5 Construction of higher-order invariants 10

$\begin{array}{lll}5.1 & \text { Number and structure of the invariants } & 10\end{array}$

5.2 Explicit construction of the invariants 13

$\begin{array}{lll}\text { 5.2.1 Needed projection operators } & 13\end{array}$

$\begin{array}{lll}5.2 .2 & \text { Algebraically independent invariants } & 14\end{array}$

$\begin{array}{ll}\text { 5.2.3 Completing the generating set } & 14\end{array}$

6 Systematic construction of Syzygies $\quad 15$

7 Necessary and sufficient conditions for explicit CPV 16

8 Towards a Hironaka decomposition 18

9 Summary and discussion $\quad 19$

$\begin{array}{ll}\text { A Algebraic (in)dependence of invariants } & 20\end{array}$

B Building blocks in conventional notation $\quad 21$

C Relation to the invariants of Gunion and Haber 22

D Explicit statement of the invariants 22

D.1 Algebraically independent invariants 22

D.2 Invariants to complete the generating set 23

$\begin{array}{ll}\text { E Syzygies } & 26\end{array}$

\section{Introduction}

Physical observables - i.e. measurable quantities like cross sections, branching ratios, CP asymmetries etc. - must not depend on arbitrary choices of basis and notation. Consequently, observables ultimately can only depend on basis invariant quantities. 
Nevertheless, in order to formulate a theory and execute computations it arguably is necessary to pick a certain basis and parametrization. Given a theory formulated in an arbitrary basis, the following questions arise:

- How does one obtain basis invariant quantities?

- How many independent basis invariant quantities exist?

- How are these basis invariant quantities related to physical observables?

This paper will make contact with all of these questions. The exemplary case treated here is the scalar potential of the two-Higgs-doublet model (2HDM), see e.g. [1]. For this case, we will be able to give definite answers to questions one and two. Moreover, physical matters will be touched with respect to the violation of the combined charge-conjugation and parity $(\mathrm{CP})$ symmetry.

Physical questions can be obscured by the presence of large basis redundancies, in which case an invariant formulation offers a clear benefit [2]. In particular - but certainly not limited to it - this is true for detecting the (non-)conservation of $\mathrm{CP}$ symmetry, which is commonly plagued by spurious phases [3]. Therefore, perhaps the most prominent example for a basis invariant measure is the well-known Jarlskog invariant, which detects CP non-conservation in the Standard Model (SM) [4].

While the non-vanishing of any CP-odd basis invariant unambiguously signals CP violation, sufficient conditions for $\mathrm{CP}$ conservation are much harder to find. That is because in order to formulate sufficient conditions one has to know "when to stop looking" for new independent invariants, which is of course at the heart of our second question above. In this light, it is no surprise that basis invariant necessary conditions for CP conservation have been formulated for theories with extended fermion [5, 6] and/or scalar sectors [7, 8], while sufficient conditions for CP conservation, besides for the SM, are only known for the 2HDM [9] (see also [10-12]) and under limiting assumptions also for the three and NHDM [12], as well as for certain supersymmetric models [13, 14]. Lately, also basis invariant necessary and sufficient conditions for the physically distinct order $4 \mathrm{CP}$ transformation of the 3HDM [15] have been formulated [16, 17].

Basis invariant methods have also been used to grasp physical aspects other than CP. For example, to investigate quark and lepton mixing in the SM and extensions [18-20], as well as to express physical observables of the 2HDM [21, 22], and more recently [23-25]. As an additional benefit, a basis invariant parametrization simplifies the analysis of renormalization group equations (RGE) and RGE running, both for SM fermions [26-28] as well as for extended scalar sectors [29-31], and so the question of how to construct basis invariants continues to be of interest [32, 33]. However, it remains in general an open question how a theory can be formulated solely in terms of basis invariant quantities.

In a group theoretical sense, basis invariants are objects which do not transform under the action of the group of basis changes. This implies that basis invariants form a ring, 
in the algebraic sense, and the question "when to stop looking" for new invariants turns out to be a mathematical exercise of invariant theory (see e.g. [34, 35]). The classical way of dealing with invariant rings is via their generating function, the so-called HilbertPoincaré series (HS). A more modern tool in ring theory is the Plethystic logarithm (PL), introduced in [36] and further discussed in [37]. The methodology of the HS and PL has been put forward for physics applications in $[38,39]$, and has subsequently been applied to a plethora of formal questions, see e.g. [40-46]. Important phenomenological applications of the HS method are the characterization of quark and lepton invariants in and beyond the SM [19, 20], as well as the general construction of complete bases of gauge invariant operators in effective field theories [47-49]. We recommend [50] as a first read on HS in the particle physics context.

In this paper, we will use the HS and PL to find the number of independent basis invariants, a generating set of basis invariants, as well as the structure of interrelations between basis invariant (syzygies) - all for the 2HDM.

The main original aspect of the present paper is our novel way of explicitly constructing the basis invariants in a systematic way. Of course, the construction of invariants from covariant objects is, in principle, a solved group theoretical problem for which even powerful computer codes exist (see e.g. [51]). However, available methods quickly become unmanageable if it comes to construction of singlets from high-rank tensors or disentangling individual contributions of, in principle, independent singlets. Our approach is to use hermitian projection operators [52] (see also [53-55]), which can conveniently be constructed from Young tableaux via birdtrack diagrams $[56,57]$. As a pedagogical introduction to this we recommend [58]. These operators project arbitrary rank tensors onto their contained orthogonal trivial singlets, implying that they give rise to the shortest possible invariants by construction. Ultimately, it is the liaison of group theoretical and algebraic techniques that merges in a new and powerful systematic way for the construction of basis invariants. The thereby constructed invariants are short and their relations transparent, as demonstrated by our new and simple proof of necessary and sufficient conditions for explicit CP conservation in the $2 \mathrm{HDM}$.

The outline of the paper is as follows. In the subsequent section we will give a brief synopsis of indispensable terms and concepts. In section 3 we turn to the $2 \mathrm{HDM}$, for which we construct the elementary building blocks of all invariants from the coupling tensors of the Lagrangian. The CP transformation behavior of the building blocks and higherorder invariants is derived in section 4. Using the building blocks as input, we proceed to construct the Hilbert series and Plethystic logarithm of the ring in section 5.2, thereby gaining information on the number and structure of the invariants and their syzygies. The explicit construction of the invariants is performed in section 5.2.2 and section 5.2.3, and a generally applicable strategy for the construction of syzygies is outlined in section 6 . We then give a simple derivation of the necessary and sufficient conditions for explicit CP conservation in the $2 \mathrm{HDM}$ in section 7 . Finally, section 8 contains some comments on the construction of a Hironaka decomposition of the $2 \mathrm{HDM}$ ring, after which we conclude. 


\section{Synopsis of jargon}

Before starting the actual discussion of this paper let us introduce the technical terms used. All parameters of a theory do transform in some way or another under necessarily unphysical changes of basis. Thus, it is in general possible to form combinations of parameters which are unaffected by all possible basis transformations: basis invariants (or short invariants in the following). Since any sum or product of invariants is an invariant itself, the invariants form a ring in the mathematical sense. ${ }^{1}$ An immediate question then is: how many invariant quantities are needed, in general, in order to be able to cover the space of all possible invariant quantities of a given model.

Some remarks are in order concerning the use of "generating set", "generators", "independent" and so forth since most of the physics literature lacks scrutiny in this point. By "independent" we always mean algebraically independent. To be clear, an invariant, say $\mathcal{I}_{1}$, is algebraically dependent on a set of invariants, say $\mathcal{I}_{2,3, . .}$, if and only if it is possible to find a polynomial $P$ such that

$$
P\left(\mathcal{I}_{1}, \mathcal{I}_{2}, \mathcal{I}_{3}, \ldots\right)=0 \text {. }
$$

If such a polynomial does not exist, $\mathcal{I}_{1}$ is called algebraically independent of $\mathcal{I}_{2,3, \ldots}$. The maximal number of algebraically independent invariants is equal to the number of physical parameters of a theory in the usual sense. Not surprisingly, this is the number of parameters which remains after all possible basis changes have been used to absorb parameters, i.e. set as many of them to zero as possible. Thus, having found a full set of algebraically independent invariants, the physical content of a theory is fully specified. Nevertheless, for many applications it makes sense to go beyond the set of algebraically independent invariants. This is the case, particularly because a relation of the kind (2.1) does not guarantee that we can solve for an arbitrary invariant. For this reason, it makes sense to discuss a generating set of a ring, which consists of all invariants that cannot be written as a polynomial of other invariants,

$$
\mathcal{I}_{i} \neq P\left(\mathcal{I}_{j}, \ldots\right) .
$$

More intuitively perhaps, the generating set of invariants is a set such that all invariants in the ring can be written as polynomial in the generators,

$$
\mathcal{I}=P\left(\mathcal{I}_{1}, \mathcal{I}_{2} \ldots\right)
$$

A classic result is that the generating set of a ring of invariants has finite size ("the ring is finitely generated") if the underlying group of symmetry transformations (in our case the group of basis transformations) is reductive [59-61]. ${ }^{2}$ General formulae for the number of invariants in the generating set are not available, but there exist bounds $[62,63]$ (see also [19] for applications).

The full set of generators of a ring is, generally, algebraically dependent (otherwise the ring is called free). Algebraic relations among invariants are called syzygies.

\footnotetext{
${ }^{1}$ Most likely the invariants form even more than a ring, since one may also envisage more involved operations on them.

${ }^{2}$ All finite groups and all semi-simple Lie groups are reductive.
} 


\section{Construction of the building blocks}

The most general 2HDM scalar potential can be written as

$$
V=\Phi_{a}^{\dagger} Y_{b}^{a} \Phi^{b}+\Phi_{a}^{\dagger} \Phi_{b}^{\dagger} Z_{c d}^{a b} \Phi^{c} \Phi^{d}
$$

where $\Phi^{a}$ are hyper charge-one scalar doublets of the $\mathrm{SU}(2)_{\mathrm{L}}$ gauge symmetry and $a, b, c, d=$ 1,2 are indices in the $\mathrm{SU}(2)$ space of Higgs-flavor. We use upper and lower indices to distinguish fields transforming as $\mathbf{2}$ and $\overline{\mathbf{2}}$ under SU(2) Higgs-flavor basis changes.

Due to hermiticity of the Lagrangian, the coupling tensors satisfy

$$
Y_{b}^{a}=\left(Y_{a}^{b}\right)^{*}, \quad Z_{c d}^{a b}=\left(Z_{a b}^{c d}\right)^{*}
$$

Because of $\mathrm{SU}(2)_{\mathrm{L}}$ invariance the quartic couplings fulfill in addition

$$
Z_{c d}^{a b}=Z_{d c}^{b a}
$$

Due to these constraints, not all entries of the coupling tensors are independent. $Y$ and $Z$ have 4 and 10 independent real entries, respectively. Utilizing all possible basis changes to absorb parameters (i.e. set them to zero) one can show that the number of physical parameters is 11 . We will, however, keep working in a general basis.

Our first goal is to construct linear combinations of the entries of $Y$ and $Z$ which transform in irreducible representations under basis changes. In particular we will see that $Y$ and $Z$ decompose into

$$
\begin{aligned}
& Y \hat{=} \mathbf{1} \oplus \mathbf{3}, \\
& Z \widehat{=} \mathbf{1} \oplus \mathbf{1} \oplus \mathbf{3} \oplus \mathbf{5} .
\end{aligned}
$$

There are three "trivial" basis invariants which arise as linear combinations of the potential parameters. Linearly independent of these, there are other linear combinations of entries of $Y$ and $Z$ which transform covariantly under basis changes, and they will be our building blocks for the construction of non-linear higher-order invariants below.

Let us be very explicit in deriving (3.4). The undertaken steps may seem like an overkill to advanced readers, but it seems necessary to recall these details before entering the derivation of higher-order invariants below.

As we want to make use of Young tableaux, it makes sense to convert all indices to one type (upper or lower). To do this, we use the fully anti-symmetric Levi-Civita tensor with the convention $\varepsilon^{12}=-\varepsilon^{21}=-\varepsilon_{12}=\varepsilon_{21}=1$ to raise or lower indices, thereby defining the objects

$$
Y^{a b}:=\varepsilon^{b c} Y_{c}^{a}, \quad \text { and } \quad Z^{a b, c d}:=\varepsilon^{c e} \varepsilon^{d f} Z_{e f}^{a b}
$$


Assigning a box to each index in the usual way, we can decompose $Y$ and $Z$ into their covariantly transforming irreducible components by the standard procedure, ${ }^{3}$

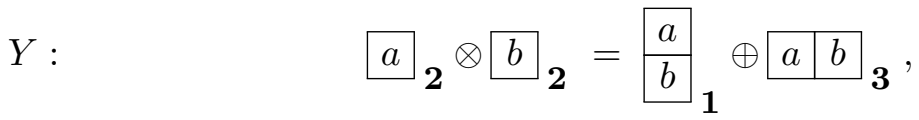



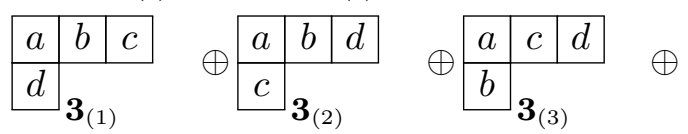

$$
\begin{aligned}
& \begin{array}{|l|l|l|l|}
\hline a & b & c & d \\
\hline
\end{array} \text {. }
\end{aligned}
$$

Recall that writing boxes in a line of the Young tableaux means symmetrization of the respective indices, while boxes in the same column are to be anti-symmetrized. The order of indices has to be obeyed. The (anti-)symmetrization of indices can be performed by projection operators. Thus, in their very essence, Young tableaux correspond to projection operators. These projection operators can always be made hermitian [52]. Acting with these projection operators on a corresponding tensor projects out the respective covariantly transforming components of the tensor. Birdtracks $[56,57]$ can be used to construct the projection operators by hand. A pedagogical introduction to this can be found in [58]. We will only recall the very most relevant features along the way.

The simplest projection operators are the total (anti-)symmetrizers of two indices denoted by

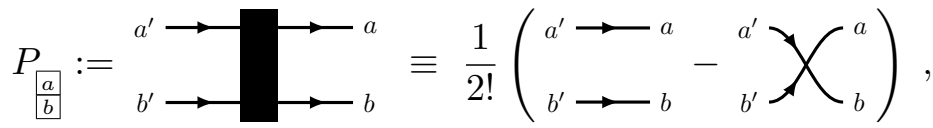

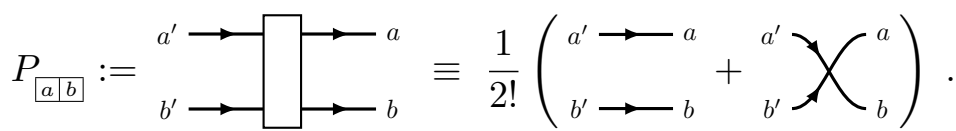

Indices on incoming(outgoing) lines correspond to upper(lower) indices, and if two indices are directly connected by a line they are meant to be set equal by contraction with a Kronecker delta. Using only these and larger (anti-)symmetrization operators consecutively, it is possible to diagrammatically construct projection operators for every Young tableaux.

Let us also introduce a diagrammatic representation of the Levi-Civita symbol

$$
\begin{aligned}
& \varepsilon^{a b} \equiv a \longrightarrow \boldsymbol{\sim} b=-a \longrightarrow \boldsymbol{\sim} b, \\
& \varepsilon_{a b} \equiv a \longrightarrow \boldsymbol{\longrightarrow} b=-a \longrightarrow \boldsymbol{\nabla} b,
\end{aligned}
$$

with the usual identities



\footnotetext{
${ }^{3}$ Due to the constraints (3.2) and (3.3), only one of the three triplets $\mathbf{3}_{(1,2,3)}$ in the decomposition of $Z$ is independent, such that eq. (3.4) holds. We will show this explicitly around eq. (3.24).
} 
It is very important to note that for indices in the fundamental of $\mathrm{SU}(N)$ we can antisymmetrize at most $N$ indices, otherwise the result is trivially zero. Diagrammatically, this gives rise to a factorization rule for anti-symmetrization operators with $N$ indices. Here, for $N=2:^{4}$

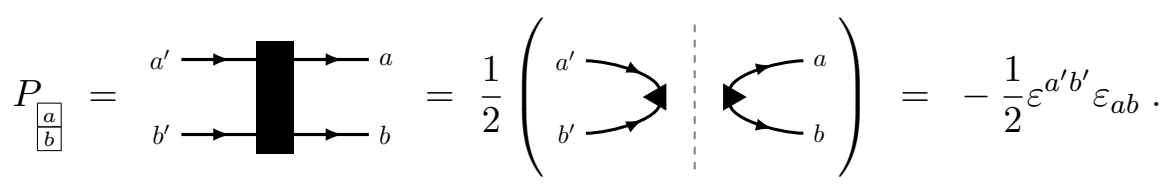

Finally, let us also introduce a diagrammatic notation for $Y$ and $Z$,

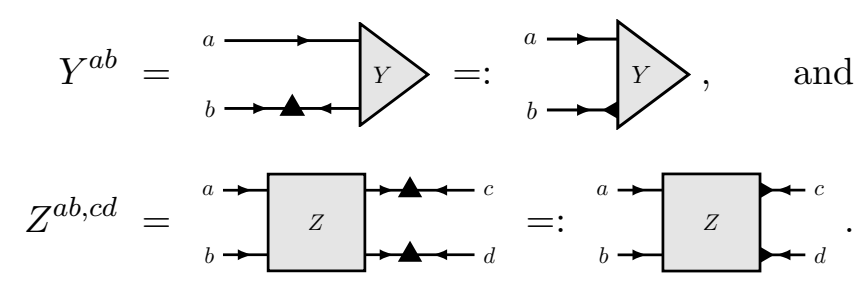

To find the covariantly transforming components of the tensor $Y$, we simply act on it with the corresponding projection operators,

$$
Y_{\mathbf{1}}^{a^{\prime} b^{\prime}}=\left[P_{\left[\frac{a}{b}\right]}\right]^{a^{\prime} b^{\prime}}{ }_{a b} Y^{a b}, \quad \text { and } \quad Y_{\mathbf{3}}^{a^{\prime} b^{\prime}}=\left[P_{[a] b]}\right]_{a b}^{a^{\prime} b^{\prime}} Y^{a b}
$$

Diagrammatically the first projection reads

$$
Y_{1}^{a^{\prime} b^{\prime}}=\frac{1}{2}{ }_{b^{\prime}}^{a^{\prime}} \longrightarrow Y=\frac{1}{2}{ }_{b^{\prime}}^{a^{\prime}} \longrightarrow
$$

Note how due to factorization of the projection operator a bubble diagram is nucleated. Clearly, $Y_{1}^{a^{\prime} b^{\prime}}$ is a basis invariant because $\varepsilon^{a^{\prime} b^{\prime}}$ is invariant under any $\mathrm{SU}(2)$ rotation while $Y_{a}^{a}$ is fully contracted. We are just interested in the non-trivial essence of the invariant. Hence, the global prefactors as well as the remaining $\varepsilon$-tensor are irrelevant and we will just drop them. The first basis invariant hence is



Factorization does not take place for the second projection with the symmetrizer, which results in

$$
Y_{\mathbf{3}}^{a^{\prime} b^{\prime}}:=\stackrel{a^{\prime} \longrightarrow \longrightarrow}{b^{\prime} \rightarrow \longrightarrow}=\frac{1}{2}\left(Y^{a^{\prime} b^{\prime}}+Y^{b^{\prime} a^{\prime}}\right) \text {. }
$$

\footnotetext{
${ }^{4}$ Spelled out as $\delta^{a^{\prime}}{ }_{a} \delta^{b^{\prime}}{ }_{b}-\delta^{a^{\prime}}{ }_{b} \delta^{b^{\prime}}{ }_{a}=-\varepsilon^{a^{\prime} b^{\prime}} \varepsilon_{a b}$, this is nothing but the well-known Schouten identity for $\mathrm{SU}(2)$ with two indices lowered (see e.g. [64]). The crucial point here is really that this leads to (in some cases only partly) factorization of this and much larger projection operators (see below), also generalizing to $\mathrm{SU}(N)$.
} 
The hermitian projection operators needed to decompose $Z$ are more involved. They can be constructed following the rules of $[53-55] .{ }^{5}$ The projectors for the singlets read

$$
P_{\frac{a b b}{c c d}}=\frac{4}{3}
$$

while the triplet projection operators are given by

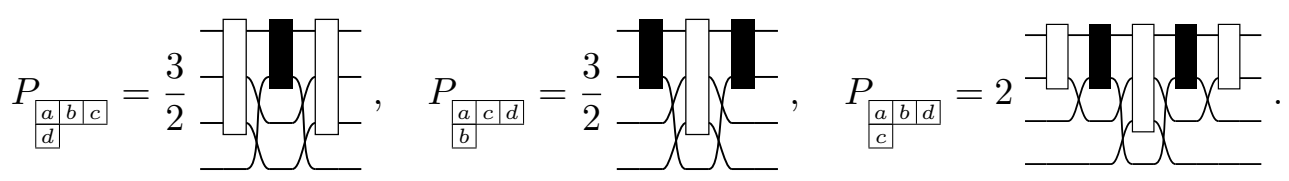

Here and in the following we will drop arrows and indices on birdtracks whenever restoring them works in the obvious way. The last remaining projection operator is $P_{\mathbf{5}}$ to project on \begin{tabular}{|l|l|l|l|l|l}
\hline$a$ & $b$ & $c$ & $d$
\end{tabular} . This is simply the total symmetrizer of four indices, and so we do not display it.

Again, note how due to the factorization rule (3.12), both of the singlet projection operators in (3.19) factorize. Upon projection this nucleates a vacuum bubble diagram which transforms as a trivial singlet. Acting with the operators of eq. (3.19) on $Z$, the two singlets result as

$$
Z_{\mathbf{1}_{(1)}}:=
$$

Next we construct the triplet building blocks of $Z$. However, before applying projection operators on $Z$ to extract the triplet irreps, observe that due to the symmetry in eq. (3.3), $Z$ identically decomposes into

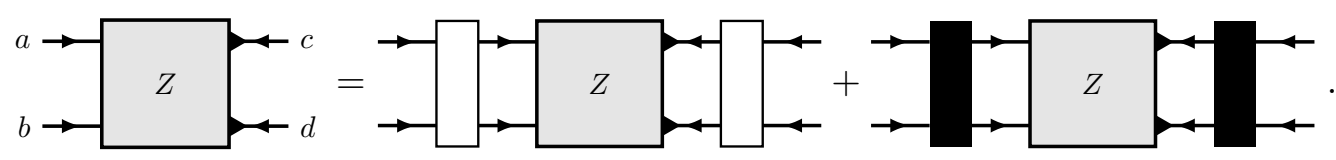

The second term is immediately identified as the trivial singlet $Z_{\mathbf{1}_{(2)}}$ by using the factorization rule (3.12). Hence, it will not contribute to the triplet irreps. Using this decomposition it is also easy to see that the triplet $\mathbf{3}_{(2)}$ vanishes identically: the corresponding projection operator (in the middle of (3.20)) anti-symmetrizes the first two indices and symmetrizes the last two indices. Thus, acting with it on $Z$ (using the decomposition (3.23)) it is

\footnotetext{
${ }^{5}$ While the rules in [53-55] are derived with mathematical rigor, following them to construct projection operators in practice can be tedious. We remark here that there is a set of simple and very intuitive rules to construct these (and much larger) projection operators from scratch. We plan to communicate these rules in the future.
} 
unavoidable that symmetrizers and anti-symmetrizers get mutually connected - which always annihilates any contribution. The remaining two triplets, $\mathbf{3}_{(1)}$ and $\mathbf{3}_{(3)}$ of eq. (3.7), are degenerate. This can be shown via a straightforward direct computation, or alternatively diagrammatically. We find

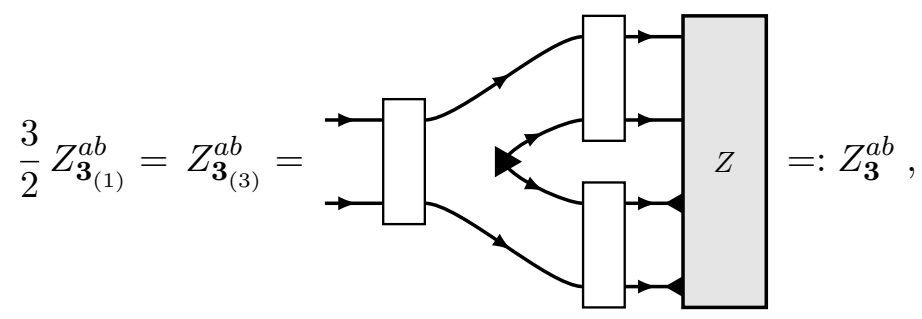

where we have deformed the box of $Z$ to make the result more pleasant to the eye. Spelling out $Z_{\mathbf{3}}^{a b}$ explicitly would already be cumbersome, while diagrammatically it can be represented in a compact way.

Finally, the five-plet $Z_{\mathbf{5}}$ is simply given by the total symmetrization of all indices of $Z$ and so we do not show this.

To conclude their construction, we explicitly state the obtained building blocks in terms of the components of $Y$ and $Z$. Denoting their components as $[Y]_{b}^{a}=y_{a b}$ and $[Z]_{c d}^{a b}=z_{a b c d}$, and dropping the irrelevant global prefactors of all covariants, one finds

$$
\begin{aligned}
Y_{\mathbf{1}} & =y_{11}+y_{22}, \\
Z_{\mathbf{1}_{(1)}} & =z_{1111}+z_{1212}+z_{1221}+z_{2222}, \\
Z_{\mathbf{1}_{(2)}} & =z_{1212}-z_{1221}, \\
Y_{\mathbf{3}}^{a b} & =\left(\begin{array}{cc}
y_{12} & \frac{1}{2}\left(y_{22}-y_{11}\right) \\
\frac{1}{2}\left(y_{22}-y_{11}\right) & -y_{12}^{*}
\end{array}\right), \\
Z_{\mathbf{3}}^{a b} & =\left(\begin{array}{cc}
z_{1112}+z_{1222} & \frac{1}{2}\left(z_{2222}-z_{1111}\right) \\
\frac{1}{2}\left(z_{2222}-z_{1111}\right) & -\left(z_{1112}+z_{1222}\right)^{*}
\end{array}\right), \\
Z_{\mathbf{5}}^{a b c d}= & \left.\left(\begin{array}{cc}
\zeta_{1} & \zeta_{2} \\
\zeta_{2} & \zeta_{3} \\
\zeta_{2} & \zeta_{3} \\
\zeta_{3} & -\zeta_{2}^{*}
\end{array}\right)\left(\begin{array}{rr}
\zeta_{2} & \zeta_{3} \\
\zeta_{3} & -\zeta_{2}^{*} \\
\zeta_{3} & -\zeta_{2}^{*} \\
-\zeta_{2}^{*} & \zeta_{1}^{*}
\end{array}\right)\right) .
\end{aligned}
$$

Here, $\left\{y_{11}, y_{22}, z_{1111}, z_{1212}, z_{1221}, z_{2222}\right\} \in \mathbb{R},\left\{y_{12}, z_{1122}, z_{1222}, z_{1112}\right\} \in \mathbb{C}$, and

$$
\begin{aligned}
\zeta_{1} & :=z_{1122}, \\
\zeta_{2} & :=\frac{1}{2}\left(z_{1222}-z_{1112}\right), \\
\zeta_{3} & :=\frac{1}{6}\left(z_{1111}-2 z_{1212}-2 z_{1221}+z_{2222}\right) .
\end{aligned}
$$

This explicitly shows how the 14 independent parameters of $Y$ and $Z$ combine to form the irreps of eq. (3.4). For completeness, we have also stated the building blocks in the conventional parametrization of the $2 \mathrm{HDM}$ scalar potential in appendix B. 


\section{CP properties of the building blocks}

Having the complete set of building blocks at hand, let us study their transformation behavior under general CP symmetries. Under a general CP transformation the scalars transform as ${ }^{6}$

$$
\Phi^{a} \mapsto \Phi_{b}^{*}\left[U^{\mathrm{T}}\right]_{a}^{b}, \quad \quad \Phi_{a}^{*} \mapsto\left[U^{*}\right]_{b}^{a} \Phi^{b} .
$$

Applied to (3.1) we see that this transformation can equivalently be described by a mapping of parameters

$$
\begin{aligned}
Y_{b}^{a} & \mapsto\left[U^{\mathrm{T}} Y^{\mathrm{T}} U^{*}\right]_{b}^{a}, \\
Z_{c d}^{a b} & \mapsto\left[U^{\mathrm{T}}\right]_{a^{\prime}}^{a}\left[U^{\mathrm{T}}\right]_{b^{\prime}}^{b}\left[Z^{\mathrm{T}}\right]^{a^{\prime} b^{\prime}}{ }_{c^{\prime} d^{\prime}}\left[U^{*}\right]^{c^{\prime}}{ }_{c}\left[U^{*}\right]^{d^{\prime}} .
\end{aligned}
$$

Assuming $U$ to be symmetric ${ }^{7}$ one can always choose a basis in flavor space in which $U \propto \mathbb{1}$. Assuming for a minute that we had transformed the theory to such a basis, the transformation of the coupling tensors simplifies to

$$
\begin{gathered}
Y_{b}^{a} \mapsto Y_{a}^{b}=\left(Y_{b}^{a}\right)^{*}, \\
Z_{c d}^{a b} \mapsto Z_{a b}^{c d}=\left(Z_{c d}^{a b}\right)^{*},
\end{gathered}
$$

where we have used hermiticity, cf. (3.2), for the last equalities. For the building blocks this implies the transformation

$$
\begin{array}{llll}
Y_{\mathbf{1}} \mapsto Y_{\mathbf{1}}, & Z_{\mathbf{1}_{(1)}} \mapsto Z_{\mathbf{1}_{(1)}}, & Z_{\mathbf{1}_{(2)}} \mapsto Z_{\mathbf{1}_{(2)}}, \\
Y_{\mathbf{3}}^{a b} \mapsto-\left(Y_{\mathbf{3}}\right)_{a b}, & Z_{\mathbf{3}}^{a b} \mapsto-\left(Z_{\mathbf{3}}\right)_{a b}, & Z_{\mathbf{5}}^{a b c d} \mapsto\left(Z_{\mathbf{5}}\right)_{a b c d} .
\end{array}
$$

It follows that basis invariants (for which all indices have to be contracted) can at most transform with a sign under a CP transformation of order two. Furthermore, we immediately recognize a simple rule to distinguish $\mathrm{CP}$-even and CP-odd basis invariants:

$$
\begin{aligned}
& \text { A basis invariant is } \mathrm{CP}\left\{\begin{array}{c}
\text { even } \\
\text { odd }
\end{array}\right\} \text { iff it contains an } \\
& \left\{\begin{array}{c}
\text { even } \\
\text { odd }
\end{array}\right\} \text { number of triplet building blocks }\left(Y_{\mathbf{3}}, Z_{\mathbf{3}}\right)
\end{aligned}
$$

\section{Construction of higher-order invariants}

\subsection{Number and structure of the invariants}

We have identified all possible linear invariants. Our goal is now to construct non-linear (in the potential parameters) higher-order invariants out of the covariantly transforming

\footnotetext{
${ }^{6}$ We focus here on the transformation behavior in the SU(2) Higgs-flavor space, hence suppress the transformation with respect to spacetime and internal gauge symmetries.

${ }^{7}$ This implies that we restrict ourselves here to CP transformation of order two, see e.g. [65]. For higher-order $\mathrm{CP}$ transformations one has to carry through the matrix $U$, but the result is the same.
} 
building blocks $Y_{\mathbf{3}}, Z_{\mathbf{3}}$, and $Z_{\mathbf{5}}$. To construct these invariants explicitly, we will once again make use of hermitian Young projection operators. However, before we do this we first want to determine the size and structure (in terms of the building blocks) of the generating set of invariants. Even though it is conceivable that even this step can be performed diagrammatically, this seems like a rather tedious way to progress. We will ease this step by using the (multi-graded) Hilbert series [38, 39, 42] (see [50] for an accessible introduction and [19] for many examples in the particle physics context). The Hilbert series (HS) together with the Plethystic logarithm (PL) will allow us to fully characterize the ring of basis invariants and, furthermore, reveal the structure of all sought invariants. This input then will be merged with our diagrammatic approach to finally construct all required invariants explicitly.

The linear invariants are irrelevant for the construction of non-trivial higher-order invariants. We will, therefore, focus only on the non-trivially transforming building blocks in this section and add the linear invariants back in later.

We will first derive the (multi-graded) HS and PL and then discuss their information content. To ease the notation we define the symbols

$$
y \hat{=} Y_{\mathbf{3}}, \quad t \hat{=} Z_{\mathbf{3}}, \quad \text { and } q \hat{=} Z_{\mathbf{5}}
$$

We will need the character polynomials $\chi_{\boldsymbol{r}}(z)$ for the relevant $\mathrm{SU}(2)$ irreps $\boldsymbol{r}$, which are given by (see e.g. [50], appendix A.2)

$$
\begin{aligned}
& \chi_{\mathbf{3}}(z)=z^{2}+1+\frac{1}{z^{2}}, \\
& \chi_{\mathbf{5}}(z)=z^{4}+z^{2}+1+\frac{1}{z^{2}}+\frac{1}{z^{4}},
\end{aligned}
$$

as well as the plethystic exponential (PE), which is defined as (see e.g. [38, 39])

$$
\mathrm{PE}[z, x, \boldsymbol{r}]:=\exp \left(\sum_{k=1}^{\infty} \frac{x^{k} \chi_{\boldsymbol{r}}\left(z^{k}\right)}{k}\right) .
$$

Using the token variables $q, y$, and $t$ as defined above, the multi-graded HS is computed as (see e.g. [44] for the integral measure)

$$
\mathfrak{H}(q, y, t)=\frac{1}{2 \pi \mathrm{i}} \oint_{|z|=1} \frac{\mathrm{d} z}{z}\left(1-z^{2}\right) \operatorname{PE}[z, q, \mathbf{5}] \operatorname{PE}[z, y, \mathbf{3}] \operatorname{PE}[z, t, \mathbf{3}] .
$$

Performing the integration via the residue theorem we find

$$
\mathfrak{H}(q, y, t)=\frac{N(q, y, t)}{D(q, y, t)},
$$

with the numerator

$$
\begin{aligned}
N(q, y, t)= & 1+q t y+q^{2} t y+q t^{2} y+q t y^{2}+q^{2} t^{2} y+q^{2} t y^{2} \\
& +q^{3} t^{3}+q^{3} t^{2} y+q^{3} t y^{2}+q^{3} y^{3} \\
& -q^{3} t^{4} y-q^{3} t^{3} y^{2}-q^{3} t^{2} y^{3}-q^{3} t y^{4}-q^{4} t^{3} y^{2}-q^{4} t^{2} y^{3} \\
& -q^{5} t^{3} y^{2}-q^{5} t^{2} y^{3}-q^{4} t^{3} y^{3}-q^{5} t^{3} y^{3}-q^{6} t^{4} y^{4}
\end{aligned}
$$


and the denominator

$$
\begin{aligned}
D(q, y, t)= & \left(1-t^{2}\right)\left(1-y^{2}\right)(1-t y)\left(1-q^{2}\right)\left(1-q^{3}\right)\left(1-q t^{2}\right)\left(1-q y^{2}\right) \\
& \left(1-q^{2} t^{2}\right)\left(1-q^{2} y^{2}\right) .
\end{aligned}
$$

We have expanded $N$ and $D$ of $\mathfrak{H}(q, y, t)$ to a form in which the leading non-trivial term in $N$ is positive. In this form, we observe that the numerator is anti-palindromic, $N(q, y, t)=$ $-q^{6} y^{4} t^{4} N\left(q^{-1}, y^{-1}, t^{-1}\right)$.

Very important information is contained in the multi-graded PL, which is defined as ${ }^{8}$

$$
\mathrm{PL}[\mathfrak{H}(q, y, t)]:=\sum_{k=1}^{\infty} \frac{\mu(k) \ln \mathfrak{H}\left(q^{k}, y^{k}, t^{k}\right)}{k},
$$

where $\mu(k)$ is the Möbius function. Expanding the PL around zero for all variables we find

$$
\begin{aligned}
\operatorname{PL}[\mathfrak{H}(q, y, t)]= & t^{2}+t y+y^{2}+q^{2}+q t^{2}+q t y+q y^{2}+q^{3}+q t^{2} y+q^{2} t^{2}+q t y^{2}+q^{2} t y \\
& +q^{2} y^{2}+q^{2} t^{2} y+q^{2} t y^{2}+q^{3} t^{3}+q^{3} t^{2} y+q^{3} t y^{2}+q^{3} y^{3}-q^{2} t^{2} y^{2} \\
& -q^{2} t^{3} y^{2}-q^{2} t^{2} y^{3}-q^{3} t^{2} y^{2}-q^{2} t^{4} y^{2}-q^{3} t^{4} y-q^{2} t^{3} y^{3}-3 q^{3} t^{3} y^{2} \\
& -q^{2} t^{2} y^{4}-3 q^{3} t^{2} y^{3}-q^{4} t^{2} y^{2}-q^{3} t y^{4}-\mathcal{O}\left([t y q]^{9}\right)
\end{aligned}
$$

The usual, ungraded, HS can directly be obtained from the multi-graded version by equating all arguments ${ }^{9}$

$$
\mathfrak{h}(z) \equiv \mathfrak{H}(z, z, z)=\frac{1+z^{3}+4 z^{4}+2 z^{5}+4 z^{6}+z^{7}+z^{10}}{\left(1-z^{2}\right)^{4}\left(1-z^{3}\right)^{3}\left(1-z^{4}\right)} .
$$

As expected for a reductive group like $\mathrm{SU}(2)$, we can find a form of $\mathfrak{h}(z)$ in which all numerator coefficients are positive. In this form the numerator of $\mathfrak{h}(z)$ is palindromic, i.e. $N(z)=z^{10} N\left(z^{-1}\right)$. Finally, the ungraded $\mathrm{PL}$ is given by

$$
\mathrm{PL}[\mathfrak{h}(z)]=4 z^{2}+4 z^{3}+5 z^{4}+2 z^{5}+3 z^{6}-3 z^{7}-\mathcal{O}\left(z^{8}\right) .
$$

Now, let us point out the relevant information content of these functions for this study:

- The denominator of the HS in eq. (5.9) informs us about the smallest complete set of algebraically independent invariants. We read off that there are four algebraically independent invariants of order 2 , three of order 3 and one of order 4 .

- The leading positive terms of the multi-graded PL in eq. (5.8) correspond to the number and structure of invariants in the generating set of the ring. To be clear, the leading 19 terms correspond to all invariants which are needed to express any other invariant as a polynomial of them. For example, the leading $t^{2}$ term tells us that there will be one generating invariant originating from the tensor product $Z_{\mathbf{3}} \otimes Z_{\mathbf{3}}$. The second term, ty, tells us that there will be one invariant from the tensor product $Z_{\mathbf{3}} \otimes Y_{\mathbf{3}}$, etc.

\footnotetext{
${ }^{8}$ The original reference for this function seems to be [36] and it has also been discussed in [37]. It was introduced in a particle physics context in $[38,39]$.

${ }^{9}$ The HS of the $2 \mathrm{HDM}$ scalar sector has only very recently appeared in the literature for the first time, see eq. (A.5) of [30]. We do not include the linear invariants here, which explains the slight difference to [30]. Otherwise our results are in full agreement.
} 
- The leading negative terms of the multi-graded PL in eq. (5.8) cut-off the set of generating invariants. Furthermore, these terms tell us the structure and number of relations between the invariants. For example, the leading negative term, $-q^{2} y^{2} t^{2}$, of the total order 6 indicates that there is one relation between invariants of that structure. The term $-3 q^{3} t^{3} y^{2}$ tells us that there are three independent relations between invariants of that structure, etc.

There is more useful information encoded in these functions but this is the most relevant for the sake of this study.

\subsection{Explicit construction of the invariants}

\subsubsection{Needed projection operators}

After having found the number and internal structure of the non-linear invariants, we proceed with their explicit construction. Due to their symmetry properties, we can directly represent the building blocks as symmetrized boxes

$$
Y_{3} \equiv \square, \quad Z_{3} \equiv \square, \quad Z_{5} \equiv \square \square \square .
$$

Here we have introduced a color coding to tell apart boxes of the different building blocks. Arbitrary invariants of higher order are now obtained by taking tensor products of the building blocks and projecting out the invariants. It turns out that all required projection operators are very simple and always of the form

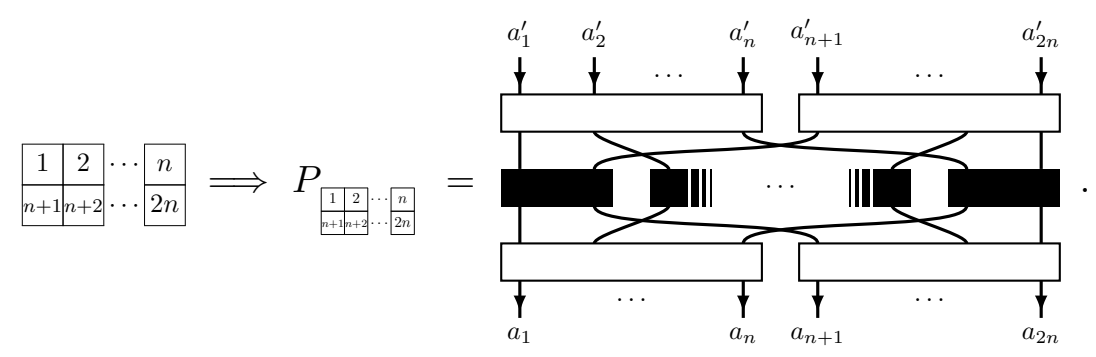

This follows from two facts: (i) We are only interested in invariants, and the operators that project onto invariants are precisely the ones which arise from Young tableaux of the "complete chocolate bar" shape (i.e. $\square$... $\boxminus$ ). (ii) Other Young tableaux of the same shape


corresponding projection operators in our case will not give rise to invariants which are independent of the ones obtained via the projection operators in (5.12).

Note that (ii) is not true in general, cf. the two independent singlets extracted from $Z$ via $\frac{12 \frac{2}{3}}{3}$ and $\frac{13}{2 \frac{3}{4}}$ above. Nonetheless, for tensor products of only $Y_{\mathbf{3}}, Z_{\mathbf{3}}$, and $Z_{\mathbf{5}}$, the statement (ii) holds and we have confirmed this explicitly. This can also be read off directly from the PL (5.8), where we find that no single invariant structure of the generating set appears with multiplicity higher than one - implying that each single tensor product $Z_{\mathbf{5}}^{\otimes a} \otimes Y_{\mathbf{3}}^{\otimes b} \otimes Z_{\mathbf{3}}^{\otimes c}$ can host at most one independent invariant. 


\subsubsection{Algebraically independent invariants}

We are now equipped to construct invariants of arbitrary order simply by projection. Let us introduce the following naming scheme for higher-order invariants:

$$
\mathcal{I}_{a, b, c} \widehat{=} \text { Invariant containing powers } q^{a}, y^{b} \text {, and } t^{c} \text {, that is } Z_{\mathbf{5}}^{\otimes a} \otimes Y_{\mathbf{3}}^{\otimes b} \otimes Z_{\mathbf{3}}^{\otimes c} \text {. }
$$

For completeness, we recall the three linear invariants, which are given by

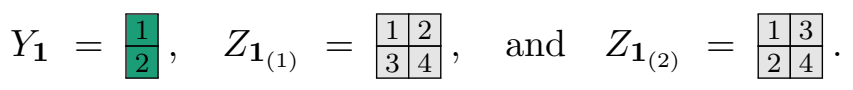

We proceed with the construction of a set of non-linear independent invariants. The order of the sought invariants corresponds to the denominator factors of the HS (5.9), and their tensor product structure can be read off from the graded PL (5.8). The resulting invariants are
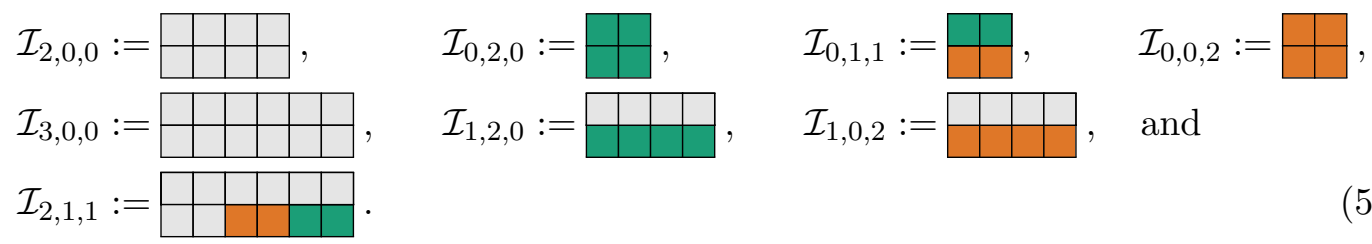

Here and in the following we suppress indices in the Young tableaux whenever they are meant to be assigned in the trivial way (incremental increase by one from left to right in each line, cf. (5.12)). The corresponding projection operators always have the form stated in (5.12), and acting with them on the respective tensor product of building blocks produces the corresponding invariant. Explicit expressions for the invariants obtained in this way are collected in appendix D and an explicit criterion to check the algebraic independence of arbitrary polynomials is given in appendix A.

Including the linear invariants we have now constructed a total of eleven invariants. These form a maximal set of algebraically independent invariants for the 2HDM. This number corresponds to the well-known number of 11 physical parameters of the $2 \mathrm{HDM}$ scalar sector. As always, the set of algebraically independent invariants is not unique. However, our choice certainly is the simplest in terms of the order of the individual invariants.

We see that for the 2HDM scalar sector it is possible to find a maximal set of algebraically independent invariants which are all CP-even. Just as in the case of the SM [19], this indicates that one can express necessary and sufficient conditions for CP conservation solely in terms of CP-even quantities. ${ }^{10}$

\subsubsection{Completing the generating set}

The complete generating set of invariants contains eleven ${ }^{11}$ additional invariants and we collect them here. After reading off their structure from the graded PL, eq. (5.8), the

\footnotetext{
${ }^{10}$ In the SM this would be the area of the CKM unitarity triangle reconstructed by the length of its sides. We thank João P. Silva for reminding us of this example.

${ }^{11}$ This number only by accident coincides with the numbers 11 above, i.e. it has nothing to do with the number of physical parameters.
} 
construction proceeds in a straightforward way. We find
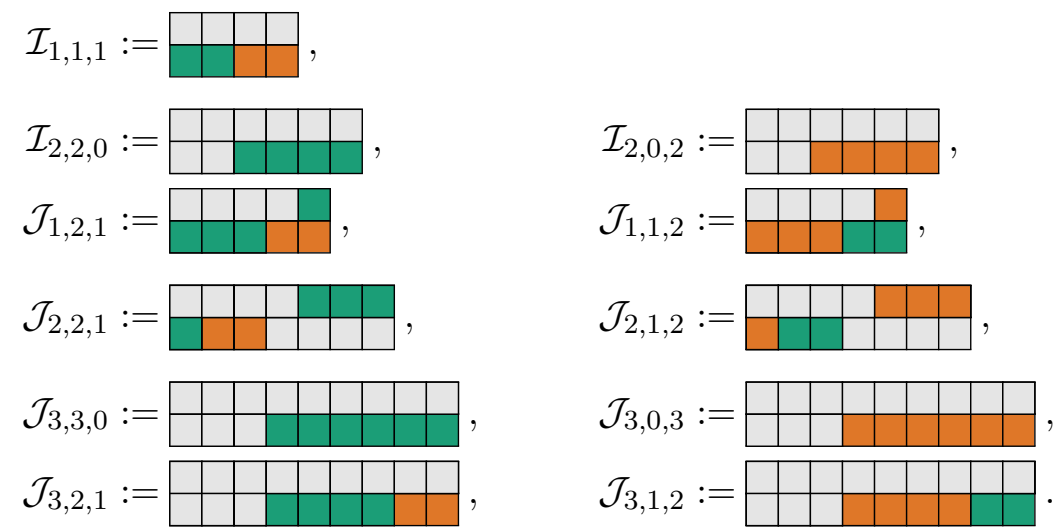

Invariants that contain an odd total number of $Y_{\mathbf{3}}$ and $Z_{\mathbf{3}}$ are CP-odd, cf. section 4 . and we denote them by the letter $\mathcal{J}$ instead of $\mathcal{I}$. We give explicit expressions for all of these invariants in appendix D. All of our invariants group into permutation representations under a $Y_{\mathbf{3}}(y) \leftrightarrow Z_{\mathbf{3}}(t)$ exchange transformation, which is, of course, what one would expect because $Y_{\mathbf{3}}$ and $Z_{\mathbf{3}}$ behave identically under basis changes.

This completes the construction of the generating set of invariants. Note that the invariants constructed in this section are not algebraically independent of the invariants in section 5.2.2 above. Therefore, each of these invariants fulfills a polynomial relation with the other invariants and we will now proceed to construct some of these relations explicitly.

\section{Systematic construction of Syzygies}

By definition of the generating set, it must be possible to express all higher-order invariants as polynomials in the invariants listed above. This requires relations between the higherorder invariants and the invariants of the generating set which are also called syzygies.

We are not aware of any previously stated generally applicable strategy to systematically construct syzygies. However, by explicit computation we find that the following strategy works here:

Any of the leading negative terms in the multi-graded PL, eq. (5.8), seems to correspond to a new, independent relation amongst the invariants. The structure of the corresponding term corresponds to the structure of the relation(s), and the coefficient of the term gives the number of independent relations of this structure. Simply making an ansatz of to-be related invariants in terms of suitable power products of lower order invariants, one just has to solve a linear system in order to obtain the desired syzygie(s).

For example, the leading negative term in (5.8) is $-q^{2} y^{2} t^{2}$. All possible power products of invariants that match this structure are

$$
\begin{array}{llll}
\mathcal{I}_{1,1,1}^{2}, & \mathcal{I}_{2,1,1} \mathcal{I}_{0,1,1}, & \mathcal{I}_{2,2,0} \mathcal{I}_{0,0,2}, & \mathcal{I}_{2,0,2} \mathcal{I}_{0,2,0}, \\
\mathcal{I}_{1,2,0} \mathcal{I}_{1,0,2}, & \mathcal{I}_{2,0,0} \mathcal{I}_{0,2,0} \mathcal{I}_{0,0,2}, & \mathcal{I}_{2,0,0} \mathcal{I}_{0,1,1}^{2} . &
\end{array}
$$


A simple linear ansatz then reveals the first syzygy: ${ }^{12}$

$$
\begin{aligned}
3 \mathcal{I}_{1,1,1}^{2}= & 2 \mathcal{I}_{2,1,1} \mathcal{I}_{0,1,1}-\mathcal{I}_{2,2,0} \mathcal{I}_{0,0,2}-\mathcal{I}_{2,0,2} \mathcal{I}_{0,2,0} \\
& +3 \mathcal{I}_{1,2,0} \mathcal{I}_{1,0,2}+\mathcal{I}_{2,0,0} \mathcal{I}_{0,2,0} \mathcal{I}_{0,0,2}-\mathcal{I}_{2,0,0} \mathcal{I}_{0,1,1}^{2}
\end{aligned}
$$

In principle it should be possible to find all syzygies by this strategy and we have explicitly checked that this works for invariants up to a total order of 14 , see table 1 .

There is a caveat: observe the differences between the (expanded) HS coefficients and the PL coefficients of the same term, for example

$$
\begin{aligned}
\mathfrak{H}(q, y, t) & =\cdots+6 q^{2} t^{2} y^{2}+\cdots+10 q^{4} t^{2} y^{2}+\ldots, \\
\operatorname{PL}[\mathfrak{H}(q, y, t)] & =\cdots-1 q^{2} t^{2} y^{2}+\cdots-1 q^{4} t^{2} y^{2}+\ldots
\end{aligned}
$$

For the above example $-q^{2} t^{2} y^{2}-$ the difference between the HS and PL coefficients $6-(-1)=7$ matches the number 7 of possible power products of the generating invariants. In some cases, however, the number of possible power products exceeds this difference. For example, this happens for the invariants of order $q^{4} t^{2} y^{2}$, for which according to the PL coefficient there is one non-trivial relation. The difference in the coefficients between HS and PL here is 11 - but we find that there are 12 possible power products. This is indicative of an additional relation of the structure $q^{4} t^{2} y^{2}$, in addition to the one counted by the " -1 " in the PL, eq. (6.3). However, this additional relation turns out not to be independent of the other relations. Rather, it is the "old" $q^{2} t^{2} y^{2}$ relation eq. (6.2) multiplied by the invariant $q^{2}$, thus producing a relation of the order $q^{4} t^{2} y^{2}$. While this happening does not give an obstacle for the explicit construction of independent syzygies, one should keep it in mind for explicit computations.

The structure of all relations that we have explicitly constructed and checked in this way are shown in table 1 in appendix E. Altogether this gives some evidence to the suspicion that one can simply read off the total number of independent relations and their structure from the negative terms of the PL.

\section{$7 \quad$ Necessary and sufficient conditions for explicit CPV}

Let us now make use of our newly gained knowledge about the 2HDMs invariants. In a formidable explicit computation [9] it has been shown that an equivalent condition to explicit $\mathrm{CP}$ conservation in the $2 \mathrm{HDM}$ scalar sector is the vanishing of the four specific CP-odd basis invariants

$$
\begin{aligned}
I_{2 Y 2 Z} & \sim \mathcal{J}_{1,2,1}, & I_{Y 3 Z} & \sim \mathcal{J}_{1,1,2}, \\
I_{3 Y 3 Z} & \sim \mathcal{J}_{3,3,0}, & I_{6 Z} & \sim \mathcal{J}_{3,0,3} .
\end{aligned}
$$

We have also stated here the corresponding invariants in the notation of this paper. " " here means that the non-trivial parts of the invariants coincide while their exact expressions

\footnotetext{
${ }^{12}$ Comparing this to the corresponding relation in the trace basis, e.g. eq. (A.4) in [30], gives a feeling for the simplification arising from the use of orthogonal projectors.
} 
may differ by a global numerical prefactor and an admixture of lower-order invariants of the correct structure. The exact relations are given in appendix C.

We will now give a very simple proof of the necessary and sufficient conditions for explicit CPV by making use of the interrelation of the invariants.

The first step of this proof is the insight that instead of a potentially infinite number of CP-odd invariants we only have to deal with the CP-odd invariants in the generating set of the ring. This is clear from the fact that any other invariant can be expressed as a polynomial in these.

From eq. (5.15) we then find that there are eight CP-odd invariants in the generating set of the ring. Thus, in order to prove Gunion and Haber right, there should be at least four independent relations amongst these invariants. In fact, we find that there are many more relations among the CP-odd invariants, cf. table 1.

The first two relations are arising at a total order 7 and they read

$$
\mathcal{J}_{2,2,1} \mathcal{I}_{0,0,2}+\mathcal{J}_{2,1,2} \mathcal{I}_{0,1,1}-\mathcal{J}_{1,2,1} \mathcal{I}_{1,0,2}+\mathcal{J}_{1,1,2} \mathcal{I}_{1,1,1}=0, \quad \text { and } \quad y \leftrightarrow t
$$

Note how this is a fully "CP-odd relation" which is actually sensitive to the signs of the CP-odd invariants and not only to their magnitude. This relation will not be used in the proof but we have stated it for completeness. Furthermore, we find two CP-odd relations of total order 8 that read

$$
3 \mathcal{J}_{2,2,1} \mathcal{I}_{1,2,0}-\mathcal{J}_{3,2,1} \mathcal{I}_{0,2,0}+3 \mathcal{J}_{3,3,0} \mathcal{I}_{0,1,1}+\mathcal{J}_{1,2,1} \mathcal{I}_{2,2,0}=0, \quad \text { and } \quad y \leftrightarrow t
$$

Finally, we also state the "CP-even" relations of the squared quintic CP-odd invariants

$$
3 \mathcal{J}_{2,2,1}^{2}+3 \mathcal{J}_{3,3,0} \mathcal{J}_{1,1,2}-\mathcal{J}_{3,2,1} \mathcal{J}_{1,2,1}-\mathcal{J}_{1,2,1}^{2} \mathcal{I}_{2,0,0}=0, \quad \text { and } \quad y \leftrightarrow t .
$$

Remarkably, the squared quintics can be expressed almost entirely in terms of products of the other CP-odd invariants.

Many more relations exist and have been derived, see table 1, but they are not needed for the proof and so we do not state them here explicitly.

Let us now show that the vanishing of the invariants in (7.1), that is

$$
\mathcal{J}_{1,2,1}=\mathcal{J}_{1,1,2}=\mathcal{J}_{3,3,0}=\mathcal{J}_{3,0,3}=0,
$$

is sufficient to conclude that all other CP-odd invariants are vanishing as well. This is readily confirmed by inspection of eqs. (7.3) and (7.4). Using (7.5) it follows from (7.4) that also $\mathcal{J}_{2,2,1}=\mathcal{J}_{2,1,2}=0$. Using this together with the condition (7.5) in (7.3) one finds that $\mathcal{J}_{3,2,1}=\mathcal{J}_{3,1,2}=0$, but only under the assumption that $\mathcal{I}_{0,2,0} \neq 0 \neq \mathcal{I}_{0,0,2}$. However, if $\mathcal{I}_{0,2,0}=\mathcal{I}_{0,0,2}=0$ were to hold, this would itself imply that $\mathcal{J}_{3,2,1}=\mathcal{J}_{3,1,2}=0$ to begin with, which is easy to show from the explicit form of the invariants given in appendix D. This completes the proof.

The in total 6 novel relations (7.2), (7.3) and (7.4) are perhaps the main results of this paper. However, many more relations of this type exist, and so we are convinced that the main use of this work is the way of how we got there. 


\section{Towards a Hironaka decomposition}

Finally, we wish to comment on the possibility of representing the ring of invariants of the $2 \mathrm{HDM}$ in an even simpler way. Due to the fact that the $\mathrm{SU}(2)$ group of basis changes is reductive, it follows that the ring of invariants obeys the Cohen-Macaulay (CM) property implying the existence of a so-called Hironaka decomposition [60, 61] (cf. also [35] section 2.3, [49] section 5.4.1, [66] appendix A, or [67] section 3).

Let us explain what that means. Writing the HS of eq. (5.9) as

$$
\mathfrak{h}(z)=\frac{\sum_{i=1}^{s} z^{s_{i}}}{\prod_{j=1}^{p}\left(1-z^{p_{j}}\right)},
$$

defines the numbers $p, p_{j}, s$, and $s_{i}{ }^{13}$ The CM property then warrants that the ring can be generated in terms of a number of $p$ primary invariants of the orders $p_{j}$, commonly denoted as $\theta_{j}$, together with a number of $s$ secondary invariants of the orders $s_{i}$, commonly denoted as $\eta_{i}$. Together, the primary and secondary invariants form a Hironaka decomposition of the ring, implying that every invariant $\mathcal{I}$ can be written as

$$
\mathcal{I}=\sum_{i=1}^{s} \eta_{i} \mathbb{C}\left[\theta_{1}, \ldots, \theta_{p}\right]
$$

Here $\mathbb{C}\left[\theta_{1}, \ldots, \theta_{p}\right]$ denotes arbitrary polynomials in the primary invariants with potentially complex coefficients and $\eta_{i}$ are the secondary invariants. The astonishing feature of this decomposition is that the secondary invariants only enter linearly.

Using the Hironaka decomposition can simplify the analysis of the ring and the related physical discussion. For example, imagine the situation that all primary invariants of a ring are $\mathrm{CP}$-even, while $\mathrm{CP}$-odd invariants only arise as secondary invariants. In order to find necessary and sufficient conditions for $\mathrm{CP}$ conservation, one then would only have to investigate the linear span of the CP-odd secondary invariants (with coefficients in the primary invariants).

We stress that the power product invariants stated above in section 5.2.2 and section 5.2.3 do not directly correspond to the primary and secondary invariants of the Hironaka decomposition. Thus, given the generating set of a ring, the main task in obtaining a Hironaka decomposition is the identification of primary and secondary invariants. This can be complicated by the fact that some of the primary and secondary invariants may only arise as combinations of invariants in the generating set. Whether this happens is dictated by the interrelations of invariants. In particular, any product of secondary invariants must either decompose in the form (8.2) or be a secondary invariant itself. The latter situation explains the appearance of secondary invariants of degree larger than any of the invariants in the generating set. These higher-order, so-called "reducible" secondary invariants can always be written as power products of a set of "irreducible" secondary invariants [68].

\footnotetext{
${ }^{13}$ For the case of eq. (5.9) one finds $p=8, s=14$ as well as the corresponding orders of the invariants $p_{j}=\{2,2,2,2,3,3,3,4\}, s_{i}=\{0,3,4,4,4,4,5,5,6,6,6,6,7,10\}$.
} 
We now comment on the construction of a Hironaka decomposition of the 2HDM ring. The primary invariants of order 2 and 3 are simply those listed in (5.14). The remaining cubic invariant $\mathcal{I}_{1,1,1}$ fulfills the relation (6.2), rendering it a bona-fide secondary invariant. The main obstacle, thus, is the identification of the lone degree 4 primary invariant. It is clear that it must be a combination of the quartic invariants given in (5.14) and (5.15). Since there are no syzygies of the structure $\left(q^{2} y^{2}\right)^{2}$, or $\left(q^{2} t^{2}\right)^{2}$, and since there are no secondary invariants of order 8 , both of the invariants $\mathcal{I}_{2,2,0}$, and $\mathcal{I}_{2,0,2}$ must be part of the sought primary invariant. In addition, the syzygy of the structure $\left(q^{2} y t\right)^{2}$ contains both, a term $\mathcal{I}_{2,1,1}^{2}$ as well as a term $\mathcal{I}_{2,2,0} \times \mathcal{I}_{2,0,2}$, thereby indicating that also the invariant $\mathcal{I}_{2,1,1}$ should appear in the sought quartic primary invariant. Other combinations of these invariants, together with the remaining quartic invariants $\mathcal{I}_{1,2,1}$ and $\mathcal{I}_{1,1,2}$ then should form the four quartic secondary invariants. Together, the sought primary and secondary invariants must obey the Hironaka decomposition (8.2). Trying to satisfy this requirement with an ansatz, however, leads to a complicated non-linear system of equations which we were unable to solve. The task of obtaining a Hironaka decomposition of the 2HDM ring, thus, remains to be solved in future work.

\section{Summary and discussion}

We have presented a new algorithm to systematically construct basis invariants. Our method is based on algebraic invariant theory, with the powerful Hilbert series and Plethystic logarithm, as well as group theory, with Young tableaux and their corresponding hermitian projection operators.

Applied to the 2HDM, we have obtained a maximal set of algebraically independent invariants, as well as a complete generating set of invariants. We have also outlined a systematic approach for the construction of syzygies and showed some of them explicitly. Using the invariants of the generating set and their syzygies we have given a concise proof of the necessary and sufficient conditions for explicit CP conservation in the 2HDM.

It should be remarked that the construction of building blocks and invariants in the 2HDM has also been performed by other means. For example, the building blocks of the present work correspond to the vectors and matrices obtained using the "bilinear formalism" [11, 69-77], and some of our CP-odd invariants are closely related to the ones obtained via graphical methods in $[9,21]$. There are some advantages of our method as compared to previous approaches. First, our strategy does not require specific basis choices at any step. Then, by the use of the powerful invariant theory functions we can with certainty identify the full set of generating invariants, their structure, as well as their interrelations. This is the first method where the answer to the question "when to stop constructing invariants" is given in a clear and quantitative fashion; it can be calculated in any model from our algorithm without the fear of miscounting. Furthermore, thanks to the use of orthogonal projection operators, our basis invariants are as short as possible by construction, and their $\mathrm{CP}$ properties are very transparent. Another attractive feature is the direct access to syzygies and their simple form of appearance. 
An immediate further application of our method to the $2 \mathrm{HDM}$ would be the inclusion of vacuum expectation values to investigate possibly realistic models and the spontaneous violation of CP. Our method also includes the possibility of identifying how the invariants behave under the various additional Higgs-flavor symmetries which are all subgroups of the group of basis changes. As a result, one would be able to identify how the number of independent invariants is reduced in models which are not the most general, but which have additional internal symmetries. Using our short invariants and relations, simplifications should also arise in the formulation of the renormalization group running [30, 31]. Another remaining open question in the $2 \mathrm{HDM}$ is the identification of combinations of invariants to obtain a Hironaka decomposition of the ring.

Perhaps more important than our explicit results on the $2 \mathrm{HDM}$ is the method itself. In principle, our strategy generalizes to the fermion sector, three or even $N$-Higgs-doublet models, and also to completely different models. A possible issue then could become computational power, since the construction of large Young projection operators easily exhausts memory limits of commercially available computing clusters. Also the computation of the Hilbert series and Plethystic logarithm straightforwardly extends to more complicated groups, but the corresponding integrals become more involved. Finally, the use of Young tableaux, at a first glance, may seem to limit our explicit construction of invariants to $\mathrm{SU}(N)$ groups. However, all that is actually needed for the systematic construction of building blocks and invariants are the hermitian projection operators for the various tensor contractions. Since birdtracks can be used to construct these for all simple Lie groups [57], we are optimistic that our method for the construction of invariants can also be generalized in this direction.

\section{Acknowledgments}

I want to thank I. P. Ivanov, C. C. Nishi, and J. P. Silva for useful discussions and motivation, and I. P. Ivanov and J. P. Silva for useful comments on the manuscript. Furthermore, I want to thank the Centre for Theoretical Particle Physics (CFTP) at Técnico Lisboa for hospitality at various stages of this work. This work has been supported by the German Science Foundation (DFG) within the SFB-Transregio TR33 "The Dark Universe". The author is grateful to the authors of the $\mathrm{IAT}_{\mathrm{E}} \mathrm{X}$ packages ytableau and youngtab, and would also like to encourage the development of packages to typeset birdtracks and birdtrack projection operators.

\section{A Algebraic (in)dependence of invariants}

The Jacobi criterion is an easy way to find the number of algebraically independent polynomials. ${ }^{14}$ For a set of polynomials (here, invariants) $\mathcal{I}_{i}$, depending on a number of variables $x_{j}$ (here, the components $y_{a b}$ and $z_{a b c d}$ of $Y$ and $Z$ ), the number of algebraically indepen-

\footnotetext{
${ }^{14}$ Strictly speaking this works only for polynomials over fields of characteristic zero, see e.g. [78, 79].
} 
dent invariants is simply given by the rank of the Jacobian matrix:

$$
\text { Number of algebraically independent invariants }=\operatorname{rank}\left[\frac{\partial \mathcal{I}_{i}}{\partial x_{j}}\right] \text {. }
$$

Besides symbolic evaluation one can also use this criterion with all variables put to random numbers for a fast machine evaluation.

\section{B Building blocks in conventional notation}

For completeness, we collect here the building blocks constructed in section 3 in the conventional parametrization of the $2 \mathrm{HDM}$ scalar potential in terms of $m$ 's and $\lambda$ 's. Following the replacement rules in ([9], eq. (21)) we find (note that their $Z_{a \bar{b} c \bar{d}} \equiv 2 Z_{b d}^{a c}$ in our notation)

$$
\begin{aligned}
y_{11} & =m_{11}^{2}, & y_{12} & =-m_{12}^{2}, & y_{22} & =m_{22}^{2}, \\
z_{1111} & =\lambda_{1} / 2, & z_{2222} & =\lambda_{2} / 2, & z_{1212} & =\lambda_{3} / 2, \quad z_{1221}=\lambda_{4} / 2, \\
z_{1122} & =\lambda_{5} / 2, & z_{1112} & =\lambda_{6} / 2 & z_{1222} & =\lambda_{7} / 2 .
\end{aligned}
$$

As usual $\left\{m_{12}^{2}, \lambda_{5}, \lambda_{6}, \lambda_{7}\right\} \in \mathbb{C}$ while all others are real. It is then straightforward to translate eq. (3.25) into this convention and we find

$$
\begin{aligned}
& Y_{1}=m_{11}^{2}+m_{22}^{2}, \\
& Z_{\mathbf{1}_{(1)}}=\frac{1}{2}\left(\lambda_{1}+\lambda_{2}+\lambda_{3}+\lambda_{4}\right), \\
& Z_{\mathbf{1}_{(2)}}=\frac{1}{2}\left(\lambda_{3}-\lambda_{4}\right), \\
& Y_{\mathbf{3}}^{a b}=\left(\begin{array}{cc}
-m_{12}^{2} & \frac{1}{2}\left(m_{22}^{2}-m_{11}^{2}\right) \\
\frac{1}{2}\left(m_{22}^{2}-m_{11}^{2}\right) & \left(m_{12}^{2}\right)^{*}
\end{array}\right), \\
& Z_{\mathbf{3}}^{a b}=\frac{1}{2}\left(\begin{array}{cc}
\lambda_{6}+\lambda_{7} & \frac{1}{2}\left(\lambda_{2}-\lambda_{1}\right) \\
\frac{1}{2}\left(\lambda_{2}-\lambda_{1}\right) & -\left(\lambda_{6}+\lambda_{7}\right)^{*}
\end{array}\right), \\
& Z_{\mathbf{5}}^{a b c d}=\left(\left(\begin{array}{rr}
\xi_{1} & \xi_{2} \\
\xi_{2} & \xi_{3} \\
\xi_{2} & \xi_{3} \\
\xi_{3} & -\xi_{2}^{*}
\end{array}\right)\left(\begin{array}{rr}
\xi_{2} & \xi_{3} \\
\xi_{3} & -\xi_{2}^{*} \\
\xi_{3} & -\xi_{2}^{*} \\
-\xi_{2}^{*} & \xi_{1}^{*}
\end{array}\right)\right) \text {, }
\end{aligned}
$$

with

$$
\xi_{1}:=\frac{1}{2} \lambda_{5}, \quad \xi_{2}:=\frac{1}{4}\left(\lambda_{7}-\lambda_{6}\right), \quad \xi_{3}:=\frac{1}{12}\left(\lambda_{1}-2 \lambda_{3}-2 \lambda_{4}+\lambda_{2}\right)
$$




\section{Relation to the invariants of Gunion and Haber}

Here we state the original set of necessary and sufficient CP-odd invariants of Gunion and Haber ([9], eqs. (23)-(26)) in the notation of this paper,

$$
\begin{aligned}
I_{Y 3 Z} & =\operatorname{Im}\left[Z^{a i}{ }_{i c} Z^{e j}{ }_{j b} Z^{b c}{ }_{e d} Y_{a}^{d}\right]=-2 \mathrm{i} \mathcal{J}_{1,1,2}, \\
I_{2 Y 2 Z} & =\operatorname{Im}\left[Y_{b}^{a} Y_{d}^{c} Z^{b d}{ }_{a f} Z^{f i}{ }_{i c}\right]=-2 \mathrm{i} \mathcal{J}_{1,2,1}, \\
I_{6 Z} & =\operatorname{Im}\left[Z_{b d}^{a c} Z^{b \ell}{ }_{\ell f} Z^{d p}{ }_{p h} Z^{f j}{ }_{a k} Z^{k m}{ }_{j n} Z^{n h}{ }_{m c}\right]=-2 \mathrm{i} \mathcal{J}_{3,0,3}, \\
I_{3 Y 3 Z} & =\operatorname{Im}\left[Z^{a b}{ }_{c d} Z^{c d}{ }_{e g} Z^{e f}{ }_{h q} Y_{a}^{g} Y_{b}^{h} Y_{f}^{q}\right] \\
& =2 \mathrm{i} \mathcal{J}_{3,3,0}+2 \mathrm{i} \mathcal{J}_{1,2,1} \mathcal{I}_{0,1,1}+\mathrm{i} Y_{1}^{2} \mathcal{J}_{1,1,2} .
\end{aligned}
$$

The first three invariants only differ by a global numerical factor, while the fourth invariant of [9] actually contains admixtures of lower lying invariants. Note also that our invariants are pure complex numbers by construction. That is, they directly correspond to the imaginary, CP-odd part of a given contraction.

\section{Explicit statement of the invariants}

\section{D.1 Algebraically independent invariants}

In this appendix we state explicitly our choice of a maximal set of algebraically independent basis invariants of the 2HDM. Note that these invariants are not written in any specific basis, i.e. they hold for all bases.

To ease the notation we use redefined parameters (conserving the total number of degrees of freedom, of course) which reflect the now known assignment of the Lagrangian parameters in $Y$ and $Z$ into the building blocks. Furthermore, it is convenient to split parameters into their real and imaginary parts.

In our new conventions the building blocks of eq. (3.25) read

$$
\begin{aligned}
Y_{\mathbf{1}}: & y_{\mathrm{s}} \\
Z_{\mathbf{1}_{(1)}} & : s_{1} \\
Z_{\mathbf{1}_{(2)}} & : s_{2} \\
Y_{\mathbf{3}}^{a b}: & \left(\begin{array}{cc}
\mathrm{i} y_{\mathrm{i}}+y_{\mathrm{r}} & y \\
y & \mathrm{i} y_{\mathrm{i}}-y_{\mathrm{r}}
\end{array}\right), \\
Z_{\mathbf{3}}^{a b}: & \left(\begin{array}{cc}
\mathrm{i} t_{\mathrm{i}}+t_{\mathrm{r}} & t \\
t & \mathrm{i} t_{\mathrm{i}}-t_{\mathrm{r}}
\end{array}\right), \\
Z_{\mathbf{5}}^{a b c d}: & \left(\left(\begin{array}{cc}
\mathrm{i} q_{\mathrm{i} 1}+q_{\mathrm{r} 1} \mathrm{i} q_{\mathrm{i} 2}+q_{\mathrm{r} 2} \\
\mathrm{i} q_{\mathrm{i} 2}+q_{\mathrm{r} 2} & q_{3} \\
\mathrm{i} q_{\mathrm{i} 2}+q_{\mathrm{r} 2} & q_{3} \\
q_{3} & \mathrm{i} q_{\mathrm{i} 2}-q_{\mathrm{r} 2}
\end{array}\right)\left(\begin{array}{cc}
\mathrm{i} q_{\mathrm{i} 2}+q_{\mathrm{r} 2} & q_{3} \\
q_{3} & \mathrm{i} q_{\mathrm{i} 2}-q_{\mathrm{r} 2} \\
q_{3} & \mathrm{i} q_{\mathrm{i} 2}-q_{\mathrm{r} 2} \\
\mathrm{i} q_{\mathrm{i} 2}-q_{\mathrm{r} 2} & q_{\mathrm{r} 1}-\mathrm{i} q_{\mathrm{i} 1}
\end{array}\right)\right)
\end{aligned}
$$


where now all fourteen parameters $\left\{y_{\mathrm{s}}, s_{1}, s_{2}, y, y_{\mathrm{r}}, y_{\mathrm{i}}, t, t_{\mathrm{r}}, t_{\mathrm{i}}, q_{\mathrm{r} 1}, q_{\mathrm{i} 1}, q_{\mathrm{r} 2}, q_{\mathrm{i} 2}, q_{3}\right\}$ are real. Their expressions in terms of the original parameters can easily be obtained by comparison with eq. (3.25) or eq. (B.2).

The global prefactors of invariants are irrelevant for our purpose. However, the convention for the prefactors does, of course, matter in stating syzygies such as the ones in eq. (6.2), (7.2), (7.3), or (7.4). As a convention, we adjust the global prefactor of each invariant such as to render all internal coefficients integer. This choice also makes the relative coefficients in the syzygies very simple.

Explicit expressions for the algebraically independent invariants of section 5.2.2 are

$$
\begin{aligned}
& Y_{1}:=\frac{1}{\frac{1}{2}}=\left(y_{11}+y_{22}\right) \equiv y_{s}, \\
& Z_{\mathbf{1}_{(1)}}:=\begin{array}{|l|l|}
\hline 1 & 2 \\
\hline 3 & 4
\end{array}=\left(z_{1111}+z_{1212}+z_{1221}+z_{2222}\right) \equiv s_{1}, \\
& Z_{\mathbf{1}_{(2)}}:=\begin{array}{|l|l|}
\hline 1 & 3 \\
\hline 2 & 4
\end{array}=\left(z_{1212}-z_{1221}\right) \equiv s_{2}, \\
& \mathcal{I}_{0,2,0}:=\square=\left(y^{2}+y_{\mathrm{i}}^{2}+y_{\mathrm{r}}^{2}\right), \\
& \mathcal{I}_{0,1,1}:=\square=\left(t y+t_{\mathrm{i}} y_{\mathrm{i}}+t_{\mathrm{r}} y_{\mathrm{r}}\right), \\
& \mathcal{I}_{0,0,2}:=\square=\left(t^{2}+t_{\mathrm{i}}^{2}+t_{\mathrm{r}}^{2}\right), \\
& \mathcal{I}_{2,0,0}:=\square\left[\begin{array}{|l|l|l}
\hline & &
\end{array}=\left(3 q_{3}^{2}+q_{\mathrm{i} 1}^{2}+4 q_{\mathrm{i} 2}^{2}+q_{\mathrm{r} 1}^{2}+4 q_{\mathrm{r} 2}^{2}\right),\right. \\
& \mathcal{I}_{1,2,0}:=\mathrm{H}^{\mathrm{W}} \mathrm{W}=\left[q_{3}\left(2 y^{2}-y_{\mathrm{i}}^{2}-y_{\mathrm{r}}^{2}\right)+4 y\left(q_{\mathrm{i} 2} y_{\mathrm{i}}+q_{\mathrm{r} 2} y_{\mathrm{r}}\right)+2 q_{\mathrm{i} 1} y_{\mathrm{i}} y_{\mathrm{r}}+q_{\mathrm{r} 1}\left(y_{\mathrm{r}}^{2}-y_{\mathrm{i}}^{2}\right)\right], \\
& \mathcal{I}_{1,0,2}:=\begin{array}{l|l|l}
\hline & &
\end{array}=\left[q_{3}\left(2 t^{2}-t_{\mathrm{i}}^{2}-t_{\mathrm{r}}^{2}\right)+4 t\left(q_{\mathrm{i} 2} t_{\mathrm{i}}+q_{\mathrm{r} 2} t_{\mathrm{r}}\right)+2 q_{\mathrm{i} 1} t_{\mathrm{i}} t_{\mathrm{r}}+q_{\mathrm{r} 1}\left(t_{\mathrm{r}}^{2}-t_{\mathrm{i}}^{2}\right)\right],
\end{aligned}
$$

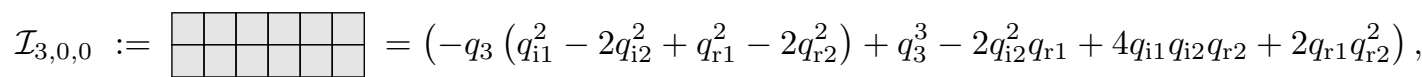

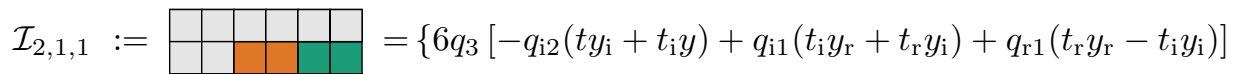

$$
\begin{aligned}
& -6 q_{\mathrm{r} 2}\left[q_{3}\left(t_{\mathrm{r}}+t_{\mathrm{r}} y\right)+q_{\mathrm{i} 1}\left(t y_{\mathrm{i}}+t_{\mathrm{i}} y\right)+2 q_{\mathrm{i} 2}\left(t_{\mathrm{i}} y_{\mathrm{r}}+t_{\mathrm{r}} y_{\mathrm{i}}\right)+q_{\mathrm{r} 1}\left(t y_{\mathrm{r}}+t_{\mathrm{r}} y\right)\right] \\
& +3 q_{3}^{2}\left(-2 t y+t_{\mathrm{i}} y_{\mathrm{i}}+t_{\mathrm{r}} y_{\mathrm{r}}\right)+6 q_{\mathrm{i} 2} q_{\mathrm{r} 1}\left(t y_{\mathrm{i}}+t_{\mathrm{i}} y\right) \\
& +q_{\mathrm{i} 1}^{2}\left(2 t y-t_{\mathrm{i}} y_{\mathrm{i}}-t_{\mathrm{r}} y_{\mathrm{r}}\right)-4 q_{\mathrm{i} 2}^{2}\left(t y+t_{\mathrm{i}} y_{\mathrm{i}}-2 t_{\mathrm{r}} y_{\mathrm{r}}\right)-6 q_{\mathrm{i} 1} q_{\mathrm{i} 2}\left(t y_{\mathrm{r}}+t_{\mathrm{r}} y\right) \\
& \left.+q_{\mathrm{r} 1}^{2}\left(2 t y-t_{\mathrm{i}} y_{\mathrm{i}}-t_{\mathrm{r}} y_{\mathrm{r}}\right)-4 q_{\mathrm{r} 2}^{2}\left(t y-2 t_{\mathrm{i}} y_{\mathrm{i}}+t_{\mathrm{r}} y_{\mathrm{r}}\right)\right\} \text {. }
\end{aligned}
$$

\section{D.2 Invariants to complete the generating set}

Here we give explicit expressions for the invariants of section 5.2.3. The statement in text-form here is provided for completeness. For practical applications we also provide the invariants in an auxiliary Mathematica notebook.

$$
\begin{aligned}
& \begin{array}{|l|l|l|}
\hline & & \\
\hline & &
\end{array}=\left[q_{3}\left(2 t y-t_{\mathrm{i}} y_{\mathrm{i}}-t_{\mathrm{r}} y_{\mathrm{r}}\right)+2 q_{\mathrm{i} 2}\left(t y_{\mathrm{i}}+t_{\mathrm{i}} y\right)\right. \\
& \left.+q_{\mathrm{i} 1}\left(t_{\mathrm{i}} y_{\mathrm{r}}+t_{\mathrm{r}} y_{\mathrm{i}}\right)+2 q_{\mathrm{r} 2}\left(t y_{\mathrm{r}}+t_{\mathrm{r}} y\right)+q_{\mathrm{r} 1}\left(t_{\mathrm{r}} y_{\mathrm{r}}-t_{\mathrm{i}} y_{\mathrm{i}}\right)\right],
\end{aligned}
$$




$$
\begin{array}{rl|l|l|}
\hline & & & \\
& & & \\
& & \mathrm{i}[ & 3 q_{3} y\left(t_{\mathrm{r}} y_{\mathrm{i}}-t_{\mathrm{i}} y_{\mathrm{r}}\right) \\
& +2 q_{\mathrm{i} 2}\left(t y y_{\mathrm{r}}-t_{\mathrm{i}} y_{\mathrm{i}} y_{\mathrm{r}}-t_{\mathrm{r}} y^{2}+t_{\mathrm{r}} y_{\mathrm{i}}^{2}\right)+q_{\mathrm{i} 1}\left(t\left(y_{\mathrm{r}}^{2}-y_{\mathrm{i}}^{2}\right)+t_{\mathrm{i}} y y_{\mathrm{i}}-t_{\mathrm{r}} y y_{\mathrm{r}}\right) \\
& +q_{\mathrm{r} 1}\left(-2 t y_{\mathrm{i}} y_{\mathrm{r}}+t_{\mathrm{i}} y y_{\mathrm{r}}+t_{\mathrm{r}} y y_{\mathrm{i}}\right) \\
& \left.+2 q_{\mathrm{r} 2}\left(-t y y_{\mathrm{i}}+t_{\mathrm{i}}\left(y-y_{\mathrm{r}}\right)\left(y+y_{\mathrm{r}}\right)+t_{\mathrm{r}} y_{\mathrm{i}} y_{\mathrm{r}}\right)\right] \\
& \\
\hline & -\mathrm{i}\left[3 q_{3} t\left(t_{\mathrm{r}} y_{\mathrm{i}}-t_{\mathrm{i}} y_{\mathrm{r}}\right)\right. \\
& +2 q_{\mathrm{i} 2}\left(t^{2} y_{\mathrm{r}}-t t_{\mathrm{r}} y+t_{\mathrm{i}}\left(t_{\mathrm{r}} y_{\mathrm{i}}-t_{\mathrm{i}} y_{\mathrm{r}}\right)\right)+q_{\mathrm{i} 1}\left(-t t_{\mathrm{i}} y_{\mathrm{i}}+t_{\mathrm{r}}\left(t y_{\mathrm{r}}-t_{\mathrm{r}} y\right)+t_{\mathrm{i}}^{2} y\right) \\
& +2 q_{\mathrm{r} 2}\left(-t^{2} y_{\mathrm{i}}+t t_{\mathrm{i}} y+t_{\mathrm{r}}\left(t_{\mathrm{r}} y_{\mathrm{i}}-t_{\mathrm{i}} y_{\mathrm{r}}\right)\right) \\
& \left.+q_{\mathrm{r} 1}\left(-t t_{\mathrm{i}} y_{\mathrm{r}}-t t_{\mathrm{r}} y_{\mathrm{i}}+2 t_{\mathrm{i}} t_{\mathrm{r}} y\right)\right],
\end{array}
$$

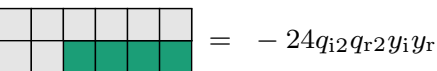

$-12\left(q_{3} q_{\mathrm{i} 2} y y_{\mathrm{i}}-q_{3} q_{\mathrm{i} 1} y_{\mathrm{i}} y_{\mathrm{r}}+q_{3} q_{\mathrm{r} 2} y y_{\mathrm{r}}-q_{\mathrm{i} 2} q_{\mathrm{r} 1} y y_{\mathrm{i}}+q_{\mathrm{i} 1} q_{\mathrm{r} 2} y y_{\mathrm{i}}+q_{\mathrm{i} 1} q_{\mathrm{i} 2} y y_{\mathrm{r}}+q_{\mathrm{r} 1} q_{\mathrm{r} 2} y y_{\mathrm{r}}\right)+8\left(q_{\mathrm{i} 2}^{2} y_{\mathrm{r}}^{2}+q_{\mathrm{r} 2}^{2} y_{\mathrm{i}}^{2}\right)$

$-6\left(q_{3} q_{\mathrm{r} 1} y_{\mathrm{i}}^{2}-q_{3} q_{\mathrm{r} 1} y_{\mathrm{r}}^{2}+q_{3}^{2} y^{2}\right)-4\left(q_{\mathrm{i} 2}^{2} y^{2}+q_{\mathrm{i} 2}^{2} y_{\mathrm{i}}^{2}+q_{\mathrm{r} 2}^{2} y^{2}+q_{\mathrm{r} 2}^{2} y_{\mathrm{r}}^{2}\right)+3\left(q_{3}^{2} y_{\mathrm{i}}^{2}+q_{3}^{2} y_{\mathrm{r}}^{2}\right)+2\left(q_{\mathrm{i} 1}^{2} y^{2}+q_{\mathrm{r} 1}^{2} y^{2}\right)$

$-q_{\mathrm{i} 1}^{2} y_{\mathrm{i}}^{2}-q_{\mathrm{i} 1}^{2} y_{\mathrm{r}}^{2}-q_{\mathrm{r} 1}^{2} y_{\mathrm{i}}^{2}-q_{\mathrm{r} 1}^{2} y_{\mathrm{r}}^{2}$

\begin{tabular}{|l|l|l|l|l|l|}
\hline & & & & & \\
\hline & & & & & \\
\hline
\end{tabular}

$+8\left(q_{\mathrm{i} 2}^{2} t_{\mathrm{r}}^{2}+q_{\mathrm{r} 2}^{2} t_{\mathrm{i}}^{2}\right)-6\left(q_{3} q_{\mathrm{r} 1} t_{\mathrm{i}}^{2}-q_{3} q_{\mathrm{r} 1} t_{\mathrm{r}}^{2}+q_{3}^{2} t^{2}\right)-4\left(q_{\mathrm{i} 2}^{2} t^{2}+q_{\mathrm{i} 2}^{2} t_{\mathrm{i}}^{2}+q_{\mathrm{r} 2}^{2} t^{2}+q_{\mathrm{r} 2}^{2} t_{\mathrm{r}}^{2}\right)+3\left(q_{3}^{2} t_{\mathrm{i}}^{2}+q_{3}^{2} t_{\mathrm{r}}^{2}\right)$

$+2\left(q_{\mathrm{i} 1}^{2} t^{2}+q_{\mathrm{r} 1}^{2} t^{2}\right)-q_{\mathrm{i} 1}^{2} t_{\mathrm{i}}^{2}-q_{\mathrm{i} 1}^{2} t_{\mathrm{r}}^{2}-q_{\mathrm{r} 1}^{2} t_{\mathrm{i}}^{2}-q_{\mathrm{r} 1}^{2} t_{\mathrm{r}}^{2}$

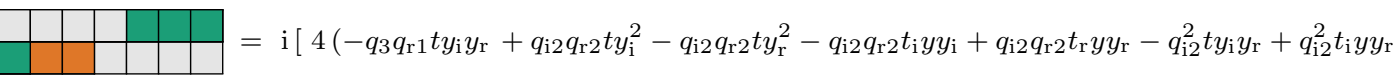

$\left.+q_{\mathrm{r} 2}^{2} t y_{\mathrm{i}} y_{\mathrm{r}}-q_{\mathrm{r} 2}^{2} t_{\mathrm{r}} y y_{\mathrm{i}}\right)+3\left(q_{3}^{2} t_{\mathrm{i}} y y_{\mathrm{r}}-q_{3}^{2} t_{\mathrm{r}} y y_{\mathrm{i}}\right)+2\left(-q_{3} q_{\mathrm{i} 2} t y y_{\mathrm{r}}-q_{3} q_{\mathrm{i} 1} t y_{\mathrm{i}}^{2}+q_{3} q_{\mathrm{i} 1} t y_{\mathrm{r}}^{2}+q_{3} q_{\mathrm{i} 1} t_{\mathrm{i}} y y_{\mathrm{i}}+q_{3} q_{\mathrm{i} 2} t_{\mathrm{i}} y_{\mathrm{i}} y_{\mathrm{r}}\right.$

$+q_{3} q_{\mathrm{i} 2} t_{\mathrm{r}} y^{2}-q_{3} q_{\mathrm{i} 1} t_{\mathrm{r}} y y_{\mathrm{r}}-q_{3} q_{\mathrm{i} 2} t_{\mathrm{r}} y_{\mathrm{i}}^{2}+q_{3} q_{\mathrm{r} 2} t y y_{\mathrm{i}}-q_{3} q_{\mathrm{r} 2} t_{\mathrm{i}} y^{2}+q_{3} q_{\mathrm{r} 1} t_{\mathrm{i}} y y_{\mathrm{r}}+q_{3} q_{\mathrm{r} 2} t_{\mathrm{i}} y_{\mathrm{r}}^{2}+q_{3} q_{\mathrm{r} 1} t_{\mathrm{r}} y y_{\mathrm{i}}-q_{3} q_{\mathrm{r} 2} t_{\mathrm{r}} y_{\mathrm{i}} y_{\mathrm{r}}$

$-q_{\mathrm{i} 1} q_{\mathrm{r} 2} t y y_{\mathrm{r}}+q_{\mathrm{i} 2} q_{\mathrm{r} 1} t y y_{\mathrm{r}}-q_{\mathrm{i} 2} q_{\mathrm{r} 1} t_{\mathrm{i}} y_{\mathrm{i}} y_{\mathrm{r}}+q_{\mathrm{i} 1} q_{\mathrm{r} 2} t_{\mathrm{i}} y_{\mathrm{i}} y_{\mathrm{r}}-q_{\mathrm{i} 2} q_{\mathrm{r} 1} t_{\mathrm{r}} y^{2}+q_{\mathrm{i} 1} q_{\mathrm{r} 2} t_{\mathrm{r}} y^{2}-q_{\mathrm{i} 1} q_{\mathrm{r} 2} t_{\mathrm{r}} y_{\mathrm{i}}^{2}+q_{\mathrm{i} 2} q_{\mathrm{r} 1} t_{\mathrm{r}} y_{\mathrm{i}}^{2}$

$\left.+q_{\mathrm{i} 1} q_{\mathrm{i} 2} t y y_{\mathrm{i}}-q_{\mathrm{i} 1} q_{\mathrm{i} 2} t_{\mathrm{i}} y^{2}+q_{\mathrm{i} 1} q_{\mathrm{i} 2} t_{\mathrm{i}} y_{\mathrm{r}}^{2}-q_{\mathrm{i} 1} q_{\mathrm{i} 2} t_{\mathrm{r}} y_{\mathrm{i}} y_{\mathrm{r}}+q_{\mathrm{r} 1} q_{\mathrm{r} 2} t y y_{\mathrm{i}}-q_{\mathrm{r} 1} q_{\mathrm{r} 2} t_{\mathrm{i}} y^{2}+q_{\mathrm{r} 1} q_{\mathrm{r} 2} t_{\mathrm{i}} y_{\mathrm{r}}^{2}-q_{\mathrm{r} 1} q_{\mathrm{r} 2} t_{\mathrm{r}} y_{\mathrm{i}} y_{\mathrm{r}}\right)$

$\left.-q_{\mathrm{i} 1}^{2} t_{\mathrm{i}} y y_{\mathrm{r}}+q_{\mathrm{i} 1}^{2} t_{\mathrm{r}} y y_{\mathrm{i}}-q_{\mathrm{r} 1}^{2} t_{\mathrm{i}} y y_{\mathrm{r}}+q_{\mathrm{r} 1}^{2} t_{\mathrm{r}} y y_{\mathrm{i}}\right]$

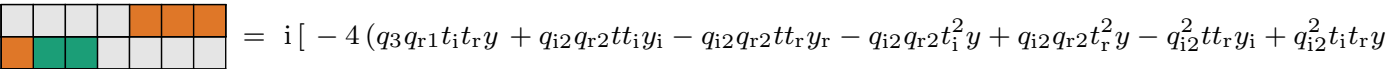

$\left.+q_{\mathrm{r} 2}^{2} t t_{\mathrm{i}} y_{\mathrm{r}}-q_{\mathrm{r} 2}^{2} t_{\mathrm{i}} t_{\mathrm{r}} y\right)-3\left(q_{3}^{2} t t_{\mathrm{i}} y_{\mathrm{r}}-q_{3}^{2} t t_{\mathrm{r}} y_{\mathrm{i}}\right)-2\left(-q_{3} q_{\mathrm{i} 2} t^{2} y_{\mathrm{r}}-q_{3} q_{\mathrm{i} 1} t t_{\mathrm{i}} y_{\mathrm{i}}+q_{3} q_{\mathrm{i} 2} t t_{\mathrm{r}} y+q_{3} q_{\mathrm{i} 1} t t_{\mathrm{r}} y_{\mathrm{r}}+q_{3} q_{\mathrm{i} 1} t_{\mathrm{i}}^{2} y\right.$

$+q_{3} q_{\mathrm{i} 2} t_{\mathrm{i}}^{2} y_{\mathrm{r}}-q_{3} q_{\mathrm{i} 2} t_{\mathrm{i}} t_{\mathrm{r}} y_{\mathrm{i}}-q_{3} q_{\mathrm{i} 1} t_{\mathrm{r}}^{2} y+q_{3} q_{\mathrm{r} 2} t^{2} y_{\mathrm{i}}-q_{3} q_{\mathrm{r} 2} t t_{\mathrm{i}} y-q_{3} q_{\mathrm{r} 1} t t_{\mathrm{i}} y_{\mathrm{r}}-q_{3} q_{\mathrm{r} 1} t t_{\mathrm{r}} y_{\mathrm{i}}+q_{3} q_{\mathrm{r} 2} t_{\mathrm{i}} t_{\mathrm{r}} y_{\mathrm{r}}-q_{3} q_{\mathrm{r} 2} t_{\mathrm{r}}^{2} y_{\mathrm{i}}$

$-q_{\mathrm{i} 1} q_{\mathrm{r} 2} t^{2} y_{\mathrm{r}}+q_{\mathrm{i} 2} q_{\mathrm{r} 1} t^{2} y_{\mathrm{r}}-q_{\mathrm{i} 2} q_{\mathrm{r} 1} t t_{\mathrm{r}} y+q_{\mathrm{i} 1} q_{\mathrm{r} 2} t t_{\mathrm{r}} y-q_{\mathrm{i} 2} q_{\mathrm{r} 1} t_{\mathrm{i}}^{2} y_{\mathrm{r}}+q_{\mathrm{i} 1} q_{\mathrm{r} 2} t_{\mathrm{i}}^{2} y_{\mathrm{r}}-q_{\mathrm{i} 1} q_{\mathrm{r} 2} t_{\mathrm{i}} t_{\mathrm{r}} y_{\mathrm{i}}+q_{\mathrm{i} 2} q_{\mathrm{r} 1} t_{\mathrm{i}} t_{\mathrm{r}} y_{\mathrm{i}}$

$\left.+q_{\mathrm{i} 1} q_{\mathrm{i} 2} t^{2} y_{\mathrm{i}}-q_{\mathrm{i} 1} q_{\mathrm{i} 2} t t_{\mathrm{i}} y+q_{\mathrm{i} 1} q_{\mathrm{i} 2} t_{\mathrm{i}} t_{\mathrm{r}} y_{\mathrm{r}}-q_{\mathrm{i} 1} q_{\mathrm{i} 2} t_{\mathrm{r}}^{2} y_{\mathrm{i}}+q_{\mathrm{r} 1} q_{\mathrm{r} 2} t^{2} y_{\mathrm{i}}-q_{\mathrm{r} 1} q_{\mathrm{r} 2} t t_{\mathrm{i}} y+q_{\mathrm{r} 1} q_{\mathrm{r} 2} t_{\mathrm{i}} t_{\mathrm{r}} y_{\mathrm{r}}-q_{\mathrm{r} 1} q_{\mathrm{r} 2} t_{\mathrm{r}}^{2} y_{\mathrm{i}}\right)+q_{\mathrm{i} 1}^{2} t t_{\mathrm{i}} y_{\mathrm{r}}$

$\left.-q_{\mathrm{i} 1}^{2} t t_{\mathrm{r}} y_{\mathrm{i}}+q_{\mathrm{r} 1}^{2} t t_{\mathrm{i}} y_{\mathrm{r}}-q_{\mathrm{r} 1}^{2} t t_{\mathrm{r}} y_{\mathrm{i}}\right]$

$=\mathrm{i}\left[18 y y_{\mathrm{i}} y_{\mathrm{r}} q_{\mathrm{r} 1} q_{3}^{2}-16\left(y_{\mathrm{i}}^{2} y_{\mathrm{r}} q_{\mathrm{i} 2} q_{\mathrm{r} 2}^{2}-y_{\mathrm{i}} y_{\mathrm{r}}^{2} q_{\mathrm{i} 2}^{2} q_{\mathrm{r} 2}\right)-12\left(y_{\mathrm{i}} q_{3} q_{\mathrm{i} 1} q_{\mathrm{i} 2} y^{2}+y_{\mathrm{r}} q_{3} q_{\mathrm{i} 2} q_{\mathrm{r} 1} y^{2}\right.\right.$

$-y_{\mathrm{r}} q_{3} q_{\mathrm{i} 1} q_{\mathrm{r} 2} y^{2}+y_{\mathrm{i}} q_{3} q_{\mathrm{r} 1} q_{\mathrm{r} 2} y^{2}-y_{\mathrm{i}} y_{\mathrm{r}} q_{3} q_{\mathrm{i} 2}^{2} y+y_{\mathrm{i}} y_{\mathrm{r}} q_{3} q_{\mathrm{r} 2}^{2} y+y_{\mathrm{i}}^{2} q_{3} q_{\mathrm{i} 2} q_{\mathrm{r} 2} y-y_{\mathrm{r}}^{2} q_{3} q_{\mathrm{i} 2} q_{\mathrm{r} 2} y+y_{\mathrm{i}}^{2} q_{\mathrm{i} 2} q_{\mathrm{r} 1} q_{\mathrm{r} 2} y$

$\left.+y_{\mathrm{r}}^{2} q_{\mathrm{i} 2} q_{\mathrm{r} 1} q_{\mathrm{r} 2} y-y_{\mathrm{i}}^{2} y_{\mathrm{r}} q_{3} q_{\mathrm{i} 2} q_{\mathrm{r} 1}-y_{\mathrm{i}} y_{\mathrm{r}}^{2} q_{3} q_{\mathrm{r} 1} q_{\mathrm{r} 2}\right)+9\left(y y_{\mathrm{i}}^{2} q_{3}^{2} q_{\mathrm{i} 1}-y y_{\mathrm{r}}^{2} q_{3}^{2} q_{\mathrm{i} 1}\right)+8\left(q_{\mathrm{i} 2} q_{\mathrm{r} 1} q_{\mathrm{r} 2} y^{3}-y_{\mathrm{r}} q_{\mathrm{i} 2}^{3} y^{2}+y_{\mathrm{i}} q_{\mathrm{r} 2}^{3} y^{2}\right.$

$\left.-y_{\mathrm{r}} q_{\mathrm{i} 2} q_{\mathrm{r} 2}^{2} y^{2}+y_{\mathrm{i}} q_{\mathrm{i} 2}^{2} q_{\mathrm{r} 2} y^{2}-y_{\mathrm{r}}^{2} q_{\mathrm{i} 1} q_{\mathrm{i} 2}^{2} y+y_{\mathrm{i}}^{2} q_{\mathrm{i} 1} q_{\mathrm{r} 2}^{2} y+y_{\mathrm{i}}^{2} y_{\mathrm{r}} q_{\mathrm{i} 2}^{3}-y_{\mathrm{i}} y_{\mathrm{r}}^{2} q_{\mathrm{r} 2}^{3}+y_{\mathrm{r}}^{3} q_{\mathrm{i} 2} q_{\mathrm{r} 2}^{2}-y_{\mathrm{i}}^{3} q_{\mathrm{i} 2}^{2} q_{\mathrm{r} 2}\right)+6\left(q_{3} q_{\mathrm{i} 1} q_{\mathrm{i} 2} y_{\mathrm{i}}^{3}\right.$

$\left.+y_{\mathrm{r}} q_{3} q_{\mathrm{i} 1} q_{\mathrm{r} 2} y_{\mathrm{i}}^{2}+y_{\mathrm{r}} q_{\mathrm{i} 1} q_{\mathrm{r} 1} q_{\mathrm{r} 2} y_{\mathrm{i}}^{2}-y_{\mathrm{r}}^{2} q_{3} q_{\mathrm{i} 1} q_{\mathrm{i} 2} y_{\mathrm{i}}+y_{\mathrm{r}}^{2} q_{\mathrm{i} 1} q_{\mathrm{i} 2} q_{\mathrm{r} 1} y_{\mathrm{i}}-y_{\mathrm{r}}^{3} q_{3} q_{\mathrm{i} 1} q_{\mathrm{r} 2}\right)+4\left(q_{\mathrm{i} 1} q_{\mathrm{i} 2}^{2} y^{3}-q_{\mathrm{i} 1} q_{\mathrm{r} 2}^{2} y^{3}+y_{\mathrm{r}} q_{\mathrm{i} 2} q_{\mathrm{r} 1}^{2} y^{2}\right.$

$+y_{\mathrm{r}} q_{\mathrm{i} 1}^{2} q_{\mathrm{i} 2} y^{2}-y_{\mathrm{i}} q_{\mathrm{i} 1}^{2} q_{\mathrm{r} 2} y^{2}-y_{\mathrm{i}} q_{\mathrm{r} 1}^{2} q_{\mathrm{r} 2} y^{2}-y_{\mathrm{i}}^{2} q_{\mathrm{i} 1} q_{\mathrm{i} 2}^{2} y+y_{\mathrm{r}}^{2} q_{11} q_{\mathrm{r} 2}^{2} y+y_{\mathrm{i}} y_{\mathrm{r}} q_{\mathrm{r} 1} q_{\mathrm{r} 2}^{2} y+y_{\mathrm{i}} y_{\mathrm{r}} q_{\mathrm{i} 2}^{2} q_{\mathrm{r} 1} y-y_{\mathrm{i}}^{2} y_{\mathrm{r}} q_{\mathrm{i} 2} q_{\mathrm{r} 1}^{2}$

$\left.+y_{\mathrm{i}} y_{\mathrm{r}}^{2} q_{\mathrm{r} 1}^{2} q_{\mathrm{r} 2}\right)-2\left(q_{\mathrm{i} 1} q_{\mathrm{i} 2} q_{\mathrm{r} 1} y_{\mathrm{i}}^{3}-q_{\mathrm{i} 1}^{2} q_{\mathrm{r} 2} y_{\mathrm{i}}^{3}-y_{\mathrm{r}} q_{\mathrm{i} 1}^{2} q_{\mathrm{i} 2} y_{\mathrm{i}}^{2}+y y_{\mathrm{r}} q_{\mathrm{r} 1}^{3} y_{\mathrm{i}}+y y_{\mathrm{r}} q_{\mathrm{i} 1}^{2} q_{\mathrm{r} 1} y_{\mathrm{i}}+y_{\mathrm{r}}^{2} q_{\mathrm{i} 1}^{2} q_{\mathrm{r} 2} y_{\mathrm{i}}+y_{\mathrm{r}}^{3} q_{\mathrm{i} 1}^{2} q_{\mathrm{i} 2}\right.$

$\left.\left.+y_{\mathrm{r}}^{3} q_{\mathrm{i} 1} q_{\mathrm{r} 1} q_{\mathrm{r} 2}\right)-y y_{\mathrm{i}}^{2} q_{\mathrm{i} 1}^{3}+y y_{\mathrm{r}}^{2} q_{\mathrm{i} 1}^{3}-y y_{\mathrm{i}}^{2} q_{\mathrm{r} 1}^{2} q_{\mathrm{i} 1}+y y_{\mathrm{r}}^{2} q_{\mathrm{r} 1}^{2} q_{\mathrm{i} 1}\right]$ 
\begin{tabular}{|l|l|l|l|l|l|l|l|l}
\hline & & & & & & & & \\
\hline & & & & & & & \\
\hline
\end{tabular}$=\mathrm{i}\left[18 t t_{\mathrm{i}} t_{\mathrm{r}} q_{\mathrm{r} 1} q_{3}^{2}-16\left(t_{\mathrm{i}}^{2} t_{\mathrm{r}} q_{\mathrm{i} 2} q_{\mathrm{r} 2}^{2}-t_{\mathrm{i}} t_{\mathrm{r}}^{2} q_{\mathrm{i} 2}^{2} q_{\mathrm{r} 2}\right)-12\left(t_{\mathrm{i}} q_{3} q_{\mathrm{i} 1} q_{\mathrm{i} 2} t^{2}+t_{\mathrm{r}} q_{3} q_{\mathrm{i} 2} q_{\mathrm{r} 1} t^{2}\right.\right.$

$-t_{\mathrm{r}} q_{3} q_{\mathrm{i} 1} q_{\mathrm{r} 2} t^{2}+t_{\mathrm{i}} q_{3} q_{\mathrm{r} 1} q_{\mathrm{r} 2} t^{2}-t_{\mathrm{i}} t_{\mathrm{r}} q_{3} q_{\mathrm{i} 2}^{2} t+t_{\mathrm{i}} t_{\mathrm{r}} q_{3} q_{\mathrm{r} 2}^{2} t+t_{\mathrm{i}}^{2} q_{3} q_{\mathrm{i} 2} q_{\mathrm{r} 2} t-t_{\mathrm{r}}^{2} q_{3} q_{\mathrm{i} 2} q_{\mathrm{r} 2} t+t_{\mathrm{i}}^{2} q_{\mathrm{i} 2} q_{\mathrm{r} 1} q_{\mathrm{r} 2} t+t_{\mathrm{r}}^{2} q_{\mathrm{i} 2} q_{\mathrm{r} 1} q_{\mathrm{r} 2} t$

$\left.-t_{\mathrm{i}}^{2} t_{\mathrm{r}} q_{3} q_{\mathrm{i} 2} q_{\mathrm{r} 1}-t_{\mathrm{i}} t_{\mathrm{r}}^{2} q_{3} q_{\mathrm{r} 1} q_{\mathrm{r} 2}\right)+9\left(t t_{\mathrm{i}}^{2} q_{3}^{2} q_{\mathrm{i} 1}-t t_{\mathrm{r}}^{2} q_{3}^{2} q_{\mathrm{i} 1}\right)+8\left(q_{\mathrm{i} 2} q_{\mathrm{r} 1} q_{\mathrm{r} 2} t^{3}-t_{\mathrm{r}} q_{\mathrm{i} 2}^{3} t^{2}+t_{\mathrm{i}} q_{\mathrm{r} 2}^{3} t^{2}-t_{\mathrm{r}} q_{\mathrm{i} 2} q_{\mathrm{r} 2}^{2} t^{2}\right.$

$\left.+t_{\mathrm{i}} q_{\mathrm{i} 2}^{2} q_{\mathrm{r} 2} t^{2}-t_{\mathrm{r}}^{2} q_{\mathrm{i} 1} q_{\mathrm{i} 2}^{2} t+t_{\mathrm{i}}^{2} q_{\mathrm{i} 1} q_{\mathrm{r} 2}^{2} t+t_{\mathrm{i}}^{2} t_{\mathrm{r}} q_{\mathrm{i} 2}^{3}-t_{\mathrm{i}} t_{\mathrm{r}}^{2} q_{\mathrm{r} 2}^{3}+t_{\mathrm{r}}^{3} q_{\mathrm{i} 2} q_{\mathrm{r} 2}^{2}-t_{\mathrm{i}}^{3} q_{\mathrm{i} 2}^{2} q_{\mathrm{r} 2}\right)+6\left(q_{3} q_{\mathrm{i} 1} q_{\mathrm{i} 2} t_{\mathrm{i}}^{3}+t_{\mathrm{r}} q_{3} q_{\mathrm{i} 1} q_{\mathrm{r} 2} t_{\mathrm{i}}^{2}\right.$

$\left.+t_{\mathrm{r}} q_{\mathrm{i} 1} q_{\mathrm{r} 1} q_{\mathrm{r} 2} t_{\mathrm{i}}^{2}-t_{\mathrm{r}}^{2} q_{3} q_{\mathrm{i} 1} q_{\mathrm{i} 2} t_{\mathrm{i}}+t_{\mathrm{r}}^{2} q_{\mathrm{i} 1} q_{\mathrm{i} 2} q_{\mathrm{r} 1} t_{\mathrm{i}}-t_{\mathrm{r}}^{3} q_{3} q_{\mathrm{i} 1} q_{\mathrm{r} 2}\right)+4\left(q_{\mathrm{i} 1} q_{\mathrm{i} 2}^{2} t^{3}-q_{\mathrm{i} 1} q_{\mathrm{r} 2}^{2} t^{3}+t_{\mathrm{r}} q_{\mathrm{i} 2} q_{\mathrm{r} 1}^{2} t^{2}+t_{\mathrm{r}} q_{\mathrm{i} 1}^{2} q_{\mathrm{i} 2} t^{2}\right.$

$\left.-t_{\mathrm{i}} q_{\mathrm{i} 1}^{2} q_{\mathrm{r} 2} t^{2}-t_{\mathrm{i}} q_{\mathrm{r} 1}^{2} q_{\mathrm{r} 2} t^{2}-t_{\mathrm{i}}^{2} q_{\mathrm{i} 1} q_{\mathrm{i} 2}^{2} t+t_{\mathrm{r}}^{2} q_{\mathrm{i} 1} q_{\mathrm{r} 2}^{2} t+t_{\mathrm{i}} t_{\mathrm{r}} q_{\mathrm{r} 1} q_{\mathrm{r} 2}^{2} t+t_{\mathrm{i}} t_{\mathrm{r}} q_{\mathrm{i} 2}^{2} q_{\mathrm{r} 1} t-t_{\mathrm{i}}^{2} t_{\mathrm{r}} q_{\mathrm{i} 2} q_{\mathrm{r} 1}^{2}+t_{\mathrm{i}} t_{\mathrm{r}}^{2} q_{\mathrm{r} 1}^{2} q_{\mathrm{r} 2}\right)$

$-2\left(q_{\mathrm{i} 1} q_{\mathrm{i} 2} q_{\mathrm{r} 1} t_{\mathrm{i}}^{3}-q_{\mathrm{i} 1}^{2} q_{\mathrm{r} 2} t_{\mathrm{i}}^{3}-t_{\mathrm{r}} q_{\mathrm{i} 1}^{2} q_{\mathrm{i} 2} t_{\mathrm{i}}^{2}+t t_{\mathrm{r}} q_{\mathrm{r} 1}^{3} t_{\mathrm{i}}+t t_{\mathrm{r}} q_{\mathrm{i} 1}^{2} q_{\mathrm{r} 1} t_{\mathrm{i}}+t_{\mathrm{r}}^{2} q_{\mathrm{i} 1}^{2} q_{\mathrm{r} 2} t_{\mathrm{i}}+t_{\mathrm{r}}^{3} q_{\mathrm{i} 1}^{2} q_{\mathrm{i} 2}+t_{\mathrm{r}}^{3} q_{\mathrm{i} 1} q_{\mathrm{r} 1} q_{\mathrm{r} 2}\right)-t t_{\mathrm{i}}^{2} q_{\mathrm{i} 1}^{3}$

$\left.+t t_{\mathrm{r}}^{2} q_{\mathrm{i} 1}^{3}-t t_{\mathrm{i}}^{2} q_{\mathrm{r} 1}^{2} q_{\mathrm{i} 1}+t t_{\mathrm{r}}^{2} q_{\mathrm{r} 1}^{2} q_{\mathrm{i} 1}\right]$

$=\mathrm{i}\left[-32\left(t_{\mathrm{i}} y_{\mathrm{i}} y_{\mathrm{r}} q_{\mathrm{i} 2} q_{\mathrm{r} 2}^{2}-t_{\mathrm{r}} y_{\mathrm{i}} y_{\mathrm{r}} q_{\mathrm{i} 2}^{2} q_{\mathrm{r} 2}\right)+24\left(t q_{\mathrm{i} 2} q_{\mathrm{r} 1} q_{\mathrm{r} 2} y^{2}-t y_{\mathrm{i}} q_{3} q_{\mathrm{i} 1} q_{\mathrm{i} 2} y-t y_{\mathrm{r}} q_{3} q_{\mathrm{i} 2} q_{\mathrm{r} 1} y\right.\right.$

$+y_{\mathrm{r}} q_{3} q_{\mathrm{i} 1} q_{\mathrm{r} 2} y-t_{\mathrm{i}} y_{\mathrm{i}} q_{3} q_{\mathrm{i} 2} q_{\mathrm{r} 2} y+t_{\mathrm{r}} y_{\mathrm{r}} q_{3} q_{\mathrm{i} 2} q_{\mathrm{r} 2} y-t y_{\mathrm{i}} q_{3} q_{\mathrm{r} 1} q_{\mathrm{r} 2} y-t_{\mathrm{i}} y_{\mathrm{i}} q_{\mathrm{i} 2} q_{\mathrm{r} 1} q_{\mathrm{r} 2} y-t_{\mathrm{r}} y_{\mathrm{r}} q_{\mathrm{i} 2} q_{\mathrm{r} 1} q_{\mathrm{r} 2} y+t_{\mathrm{r}} y_{\mathrm{r}}^{2} q_{\mathrm{i} 2} q_{\mathrm{r} 2}^{2}$ $\left.+t_{\mathrm{i}} y_{\mathrm{i}} y_{\mathrm{r}} q_{3} q_{\mathrm{i} 2} q_{\mathrm{r} 1}-t_{\mathrm{i}} y_{\mathrm{i}}^{2} q_{\mathrm{i} 2}^{2} q_{\mathrm{r} 2}+t_{\mathrm{r}} y_{\mathrm{i}} y_{\mathrm{r}} q_{3} q_{\mathrm{r} 1} q_{\mathrm{r} 2}\right)+18\left(t_{\mathrm{i}} q_{3} q_{\mathrm{i} 1} q_{\mathrm{i} 2} y_{\mathrm{i}}^{2}+t_{\mathrm{i}} y q_{3}^{2} q_{\mathrm{i} 1} y_{\mathrm{i}}+t_{\mathrm{r}} y q_{3}^{2} q_{\mathrm{r} 1} y_{\mathrm{i}}+t y_{\mathrm{r}} q_{3}^{2} q_{\mathrm{r} 1} y_{\mathrm{i}}\right.$

$\left.-t_{\mathrm{r}} y y_{\mathrm{r}} q_{3}^{2} q_{\mathrm{i} 1}+t_{\mathrm{i}} y y_{\mathrm{r}} q_{3}^{2} q_{\mathrm{r} 1}-t_{\mathrm{r}} y_{\mathrm{r}}^{2} q_{3} q_{\mathrm{i} 1} q_{\mathrm{r} 2}\right)+16\left(-t y y_{\mathrm{r}} q_{\mathrm{i} 2}^{3}+t_{\mathrm{i}} y_{\mathrm{i}} y_{\mathrm{r}} q_{\mathrm{i} 2}^{3}-t_{\mathrm{r}} y y_{\mathrm{r}} q_{\mathrm{i} 1} q_{\mathrm{i} 2}^{2}+t_{\mathrm{i}} y_{\mathrm{r}}^{2} q_{\mathrm{r} 2} q_{\mathrm{i} 2}^{2}+t y y_{\mathrm{i}} q_{\mathrm{r} 2} q_{\mathrm{i} 2}^{2}\right.$

$\left.-t_{\mathrm{r}} y_{\mathrm{i}}^{2} q_{\mathrm{r} 2}^{2} q_{\mathrm{i} 2}-t y y_{\mathrm{r}} q_{\mathrm{r} 2}^{2} q_{\mathrm{i} 2}+t y y_{\mathrm{i}} q_{\mathrm{r} 2}^{3}-t_{\mathrm{r}} y_{\mathrm{i}} y_{\mathrm{r}} q_{\mathrm{r} 2}^{3}+t_{\mathrm{i}} y y_{\mathrm{i}} q_{\mathrm{i} 1} q_{\mathrm{r} 2}^{2}\right)+12\left(t q_{\mathrm{i} 1} q_{\mathrm{i} 2}^{2} y^{2}-t q_{\mathrm{i} 1} q_{\mathrm{r} 2}^{2} y^{2}-t_{\mathrm{i}} q_{3} q_{\mathrm{i} 1} q_{\mathrm{i} 2} y^{2}\right.$

$-t_{\mathrm{r}} q_{3} q_{\mathrm{i} 2} q_{\mathrm{r} 1} y^{2}+t_{\mathrm{r}} q_{3} q_{\mathrm{i} 1} q_{\mathrm{r} 2} y^{2}-t_{\mathrm{i}} q_{3} q_{\mathrm{r} 1} q_{\mathrm{r} 2} y^{2}+t_{\mathrm{r}} y_{\mathrm{i}} q_{3} q_{\mathrm{i} 2}^{2} y+t_{\mathrm{i}} y_{\mathrm{r}} q_{3} q_{\mathrm{i} 2}^{2} y-t_{\mathrm{r}} y_{\mathrm{i}} q_{3} q_{\mathrm{r} 2}^{2} y-t_{\mathrm{i}} y_{\mathrm{r}} q_{3} q_{\mathrm{r} 2}^{2} y+t y_{\mathrm{i}} y_{\mathrm{r}} q_{3} q_{\mathrm{i} 2}^{2}$

$-t y_{\mathrm{i}} y_{\mathrm{r}} q_{3} q_{\mathrm{r} 2}^{2}-t_{\mathrm{r}} y_{\mathrm{i}} y_{\mathrm{r}} q_{3} q_{\mathrm{i} 1} q_{\mathrm{i} 2}+t_{\mathrm{r}} y_{\mathrm{i}}^{2} q_{3} q_{\mathrm{i} 2} q_{\mathrm{r} 1}+t_{\mathrm{r}} y_{\mathrm{i}} y_{\mathrm{r}} q_{\mathrm{i} 1} q_{\mathrm{i} 2} q_{\mathrm{r} 1}+t_{\mathrm{i}} y_{\mathrm{i}} y_{\mathrm{r}} q_{3} q_{\mathrm{i} 1} q_{\mathrm{r} 2}-t y_{\mathrm{i}}^{2} q_{3} q_{\mathrm{i} 2} q_{\mathrm{r} 2}+t y_{\mathrm{r}}^{2} q_{3} q_{\mathrm{i} 2} q_{\mathrm{r} 2}$

$\left.+t_{\mathrm{i}} y_{\mathrm{r}}^{2} q_{3} q_{\mathrm{r} 1} q_{\mathrm{r} 2}+t_{\mathrm{i}} y_{\mathrm{i}} y_{\mathrm{r}} q_{\mathrm{i} 1} q_{\mathrm{r} 1} q_{\mathrm{r} 2}-t y_{\mathrm{i}}^{2} q_{\mathrm{i} 2} q_{\mathrm{r} 1} q_{\mathrm{r} 2}-t y_{\mathrm{r}}^{2} q_{\mathrm{i} 2} q_{\mathrm{r} 1} q_{\mathrm{r} 2}\right)+9\left(t y_{\mathrm{i}}^{2} q_{3}^{2} q_{\mathrm{i} 1}-t y_{\mathrm{r}}^{2} q_{3}^{2} q_{\mathrm{i} 1}\right)-8\left(t_{\mathrm{r}} y^{2} q_{\mathrm{i} 2}^{3}-t_{\mathrm{r}} y_{\mathrm{i}}^{2} q_{\mathrm{i} 2}^{3}\right.$

$+t y_{\mathrm{r}}^{2} q_{\mathrm{i} 1} q_{\mathrm{i} 2}^{2}+t_{\mathrm{i}} y y_{\mathrm{i}} q_{\mathrm{i} 1} q_{\mathrm{i} 2}^{2}-t_{\mathrm{i}} y^{2} q_{\mathrm{r} 2} q_{\mathrm{i} 2}^{2}-t y y_{\mathrm{r}} q_{\mathrm{i} 1}^{2} q_{\mathrm{i} 2}-t y y_{\mathrm{r}} q_{\mathrm{r} 1}^{2} q_{\mathrm{i} 2}+t_{\mathrm{i}} y_{\mathrm{i}} y_{\mathrm{r}} q_{\mathrm{r} 1}^{2} q_{\mathrm{i} 2}+t_{\mathrm{r}} y^{2} q_{\mathrm{r} 2}^{2} q_{\mathrm{i} 2}-t_{\mathrm{i}} y^{2} q_{\mathrm{r} 2}^{3}+t_{\mathrm{i}} y_{\mathrm{r}}^{2} q_{\mathrm{r} 2}^{3}$

$\left.-t y_{\mathrm{i}}^{2} q_{\mathrm{i} 1} q_{\mathrm{r} 2}^{2}-t_{\mathrm{r}} y y_{\mathrm{r}} q_{\mathrm{i} 1} q_{\mathrm{r} 2}^{2}+t y y_{\mathrm{i}} q_{\mathrm{i} 1}^{2} q_{\mathrm{r} 2}+t y y_{\mathrm{i}} q_{\mathrm{r} 1}^{2} q_{\mathrm{r} 2}-t_{\mathrm{r}} y_{\mathrm{i}} y_{\mathrm{r}} q_{\mathrm{r} 1}^{2} q_{\mathrm{r} 2}\right)+6\left(-t_{\mathrm{i}} q_{\mathrm{i} 1} q_{\mathrm{i} 2} q_{\mathrm{r} 1} y_{\mathrm{i}}^{2}+t_{\mathrm{i}} q_{\mathrm{i} 1}^{2} q_{\mathrm{r} 2} y_{\mathrm{i}}^{2}\right.$

$\left.+t_{\mathrm{r}} q_{3} q_{\mathrm{i} 1} q_{\mathrm{r} 2} y_{\mathrm{i}}^{2}+t_{\mathrm{r}} q_{\mathrm{i} 1} q_{\mathrm{r} 1} q_{\mathrm{r} 2} y_{\mathrm{i}}^{2}-t_{\mathrm{r}} y_{\mathrm{r}}^{2} q_{\mathrm{i} 1}^{2} q_{\mathrm{i} 2}-t_{\mathrm{i}} y_{\mathrm{r}}^{2} q_{3} q_{\mathrm{i} 1} q_{\mathrm{i} 2}+t_{\mathrm{i}} y_{\mathrm{r}}^{2} q_{\mathrm{i} 1} q_{\mathrm{i} 2} q_{\mathrm{r} 1}-t_{\mathrm{r}} y_{\mathrm{r}}^{2} q_{\mathrm{i} 1} q_{\mathrm{r} 1} q_{\mathrm{r} 2}\right)-4\left(-t_{\mathrm{r}} y^{2} q_{\mathrm{i} 2} q_{\mathrm{i} 1}^{2}\right.$

$-t_{\mathrm{i}} y_{\mathrm{i}} y_{\mathrm{r}} q_{\mathrm{i} 2} q_{\mathrm{i} 1}^{2}+t_{\mathrm{i}} y^{2} q_{\mathrm{r} 2} q_{\mathrm{i} 1}^{2}+t_{\mathrm{r}} y_{\mathrm{i}} y_{\mathrm{r}} q_{\mathrm{r} 2} q_{\mathrm{i} 1}^{2}+t y_{\mathrm{i}}^{2} q_{\mathrm{i} 2}^{2} q_{\mathrm{i} 1}-t y_{\mathrm{r}}^{2} q_{\mathrm{r} 2}^{2} q_{\mathrm{i} 1}-t_{\mathrm{r}} y^{2} q_{\mathrm{i} 2} q_{\mathrm{r} 1}^{2}+t_{\mathrm{r}} y_{\mathrm{i}}^{2} q_{\mathrm{i} 2} q_{\mathrm{r} 1}^{2}-t_{\mathrm{r}} y y_{\mathrm{i}} q_{\mathrm{r} 1} q_{\mathrm{r} 2}^{2}$

$\left.-t_{\mathrm{i}} y y_{\mathrm{r}} q_{\mathrm{r} 1} q_{\mathrm{r} 2}^{2}-t y_{\mathrm{i}} y_{\mathrm{r}} q_{\mathrm{r} 1} q_{\mathrm{r} 2}^{2}-t_{\mathrm{r}} y y_{\mathrm{i}} q_{\mathrm{i} 2}^{2} q_{\mathrm{r} 1}-t_{\mathrm{i}} y y_{\mathrm{r}} q_{\mathrm{i} 2}^{2} q_{\mathrm{r} 1}-t y_{\mathrm{i}} y_{\mathrm{r}} q_{\mathrm{i} 2}^{2} q_{\mathrm{r} 1}+t_{\mathrm{i}} y^{2} q_{\mathrm{r} 1}^{2} q_{\mathrm{r} 2}-t_{\mathrm{i}} y_{\mathrm{r}}^{2} q_{\mathrm{r} 1}^{2} q_{\mathrm{r} 2}\right)-2\left(t_{\mathrm{i}} y y_{\mathrm{i}} q_{\mathrm{i} 1}^{3}\right.$

$-t_{\mathrm{r}} y y_{\mathrm{r}} q_{\mathrm{i} 1}^{3}-t_{\mathrm{r}} y_{\mathrm{i}}^{2} q_{\mathrm{i} 2} q_{\mathrm{i} 1}^{2}+t_{\mathrm{r}} y y_{\mathrm{i}} q_{\mathrm{r} 1} q_{\mathrm{i} 1}^{2}+t_{\mathrm{i}} y y_{\mathrm{r}} q_{\mathrm{r} 1} q_{\mathrm{i} 1}^{2}+t y_{\mathrm{i}} y_{\mathrm{r}} q_{\mathrm{r} 1} q_{\mathrm{i} 1}^{2}+t_{\mathrm{i}} y_{\mathrm{r}}^{2} q_{\mathrm{r} 2} q_{\mathrm{i} 1}^{2}+t_{\mathrm{i}} y y_{\mathrm{i}} q_{\mathrm{r} 1}^{2} q_{\mathrm{i} 1}-t_{\mathrm{r}} y y_{\mathrm{r}} q_{\mathrm{r} 1}^{2} q_{\mathrm{i} 1}$

$\left.\left.+t_{\mathrm{r}} y y_{\mathrm{i}} q_{\mathrm{r} 1}^{3}+t_{\mathrm{i}} y y_{\mathrm{r}} q_{\mathrm{r} 1}^{3}+t y_{\mathrm{i}} y_{\mathrm{r}} q_{\mathrm{r} 1}^{3}\right)-t y_{\mathrm{i}}^{2} q_{\mathrm{i} 1}^{3}+t y_{\mathrm{r}}^{2} q_{\mathrm{i} 1}^{3}-t y_{\mathrm{i}}^{2} q_{\mathrm{r} 1}^{2} q_{\mathrm{i} 1}+t y_{\mathrm{r}}^{2} q_{\mathrm{r} 1}^{2} q_{\mathrm{i} 1}\right]$

\begin{tabular}{l|l|l|l|l|l|l}
\hline & & & & & & \\
\hline
\end{tabular}$=\mathrm{i}\left[-32\left(t_{\mathrm{i}} t_{\mathrm{r}} y_{\mathrm{i}} q_{\mathrm{i} 2} q_{\mathrm{r} 2}^{2}-t_{\mathrm{i}} t_{\mathrm{r}} y_{\mathrm{r}} q_{\mathrm{i} 2}^{2} q_{\mathrm{r} 2}\right)+24\left(y q_{\mathrm{i} 2} q_{\mathrm{r} 1} q_{\mathrm{r} 2} t^{2}-t_{\mathrm{i}} y q_{3} q_{\mathrm{i} 1} q_{\mathrm{i} 2} t-t_{\mathrm{r}} y q_{3} q_{\mathrm{i} 2} q_{\mathrm{r} 1} t\right.\right.$

$+t_{\mathrm{r}} y q_{3} q_{\mathrm{i} 1} q_{\mathrm{r} 2} t-t_{\mathrm{i}} y_{\mathrm{i}} q_{3} q_{\mathrm{i} 2} q_{\mathrm{r} 2} t+t_{\mathrm{r}} y_{\mathrm{r}} q_{3} q_{\mathrm{i} 2} q_{\mathrm{r} 2} t-t_{\mathrm{i}} y q_{3} q_{\mathrm{r} 1} q_{\mathrm{r} 2} t-t_{\mathrm{i}} y_{\mathrm{i}} q_{\mathrm{i} 2} q_{\mathrm{r} 1} q_{\mathrm{r} 2} t-t_{\mathrm{r}} y_{\mathrm{r}} q_{\mathrm{i} 2} q_{\mathrm{r} 1} q_{\mathrm{r} 2} t+t_{\mathrm{r}}^{2} y_{\mathrm{r}} q_{\mathrm{i} 2} q_{\mathrm{r} 2}^{2}$ $\left.+t_{\mathrm{i}} t_{\mathrm{r}} y_{\mathrm{i}} q_{3} q_{\mathrm{i} 2} q_{\mathrm{r} 1}-t_{\mathrm{i}}^{2} y_{\mathrm{i}} q_{\mathrm{i} 2}^{2} q_{\mathrm{r} 2}+t_{\mathrm{i}} t_{\mathrm{r}} y_{\mathrm{r}} q_{3} q_{\mathrm{r} 1} q_{\mathrm{r} 2}\right)+18\left(y_{\mathrm{i}} q_{3} q_{\mathrm{i} 1} q_{\mathrm{i} 2} t_{\mathrm{i}}^{2}+t y_{\mathrm{i}} q_{3}^{2} q_{\mathrm{i} 1} t_{\mathrm{i}}+t_{\mathrm{r}} y q_{3}^{2} q_{\mathrm{r} 1} t_{\mathrm{i}}+t y_{\mathrm{r}} q_{3}^{2} q_{\mathrm{r} 1} t_{\mathrm{i}}\right.$ $\left.-t t_{\mathrm{r}} y_{\mathrm{r}} q_{3}^{2} q_{\mathrm{i} 1}+t t_{\mathrm{r}} y_{\mathrm{i}} q_{3}^{2} q_{\mathrm{r} 1}-t_{\mathrm{r}}^{2} y_{\mathrm{r}} q_{3} q_{\mathrm{i} 1} q_{\mathrm{r} 2}\right)+16\left(-t t_{\mathrm{r}} y q_{\mathrm{i} 2}^{3}+t_{\mathrm{i}} t_{\mathrm{r}} y_{\mathrm{i}} q_{\mathrm{i} 2}^{3}-t t_{\mathrm{r}} y_{\mathrm{r}} q_{\mathrm{i} 1} q_{\mathrm{i} 2}^{2}+t t_{\mathrm{i}} y q_{\mathrm{r} 2} q_{\mathrm{i} 2}^{2}+t_{\mathrm{r}}^{2} y_{\mathrm{i}} q_{\mathrm{r} 2} q_{\mathrm{i} 2}^{2}\right.$ $\left.-t t_{\mathrm{r}} y q_{\mathrm{r} 2}^{2} q_{\mathrm{i} 2}-t_{\mathrm{i}}^{2} y_{\mathrm{r}} q_{\mathrm{r} 2}^{2} q_{\mathrm{i} 2}+t t_{\mathrm{i}} y q_{\mathrm{r} 2}^{3}-t_{\mathrm{i}} t_{\mathrm{r}} y_{\mathrm{r}} q_{\mathrm{r} 2}^{3}+t t_{\mathrm{i}} y_{\mathrm{i}} q_{\mathrm{i} 1} q_{\mathrm{r} 2}^{2}\right)+12\left(y q_{\mathrm{i} 1} q_{\mathrm{i} 2}^{2} t^{2}-y q_{\mathrm{i} 1} q_{\mathrm{r} 2}^{2} t^{2}-y_{\mathrm{i}} q_{3} q_{\mathrm{i} 1} q_{\mathrm{i} 2} t^{2}\right.$ $-y_{\mathrm{r}} q_{3} q_{\mathrm{i} 2} q_{\mathrm{r} 1} t^{2}+y_{\mathrm{r}} q_{3} q_{\mathrm{i} 1} q_{\mathrm{r} 2} t^{2}-y_{\mathrm{i}} q_{3} q_{\mathrm{r} 1} q_{\mathrm{r} 2} t^{2}+t_{\mathrm{r}} y_{\mathrm{i}} q_{3} q_{\mathrm{i} 2}^{2} t+t_{\mathrm{i}} y_{\mathrm{r}} q_{3} q_{\mathrm{i} 2}^{2} t-t_{\mathrm{r}} y_{\mathrm{i}} q_{3} q_{\mathrm{r} 2}^{2} t-t_{\mathrm{i}} y_{\mathrm{r}} q_{3} q_{\mathrm{r} 2}^{2} t+t_{\mathrm{i}} t_{\mathrm{r}} y q_{3} q_{\mathrm{i} 2}^{2}$ $-t_{\mathrm{i}} t_{\mathrm{r}} y q_{3} q_{\mathrm{r} 2}^{2}-t_{\mathrm{i}} t_{\mathrm{r}} y_{\mathrm{r}} q_{3} q_{\mathrm{i} 1} q_{\mathrm{i} 2}+t_{\mathrm{i}}^{2} y_{\mathrm{r}} q_{3} q_{\mathrm{i} 2} q_{\mathrm{r} 1}+t_{\mathrm{i}} t_{\mathrm{r}} y_{\mathrm{r}} q_{\mathrm{i} 1} q_{\mathrm{i} 2} q_{\mathrm{r} 1}+t_{\mathrm{i}} t_{\mathrm{r}} y_{\mathrm{i}} q_{3} q_{\mathrm{i} 1} q_{\mathrm{r} 2}-t_{\mathrm{i}}^{2} y q_{3} q_{\mathrm{i} 2} q_{\mathrm{r} 2}+t_{\mathrm{r}}^{2} y q_{3} q_{\mathrm{i} 2} q_{\mathrm{r} 2}$ $\left.+t_{\mathrm{r}}^{2} y_{\mathrm{i}} q_{3} q_{\mathrm{r} 1} q_{\mathrm{r} 2}+t_{\mathrm{i}} t_{\mathrm{r}} y_{\mathrm{i}} q_{\mathrm{i} 1} q_{\mathrm{r} 1} q_{\mathrm{r} 2}-t_{\mathrm{i}}^{2} y q_{\mathrm{i} 2} q_{\mathrm{r} 1} q_{\mathrm{r} 2}-t_{\mathrm{r}}^{2} y q_{\mathrm{i} 2} q_{\mathrm{r} 1} q_{\mathrm{r} 2}\right)+9\left(t_{\mathrm{i}}^{2} y q_{3}^{2} q_{\mathrm{i} 1}-t_{\mathrm{r}}^{2} y q_{3}^{2} q_{\mathrm{i} 1}\right)+8\left(t_{\mathrm{i}}^{2} y_{\mathrm{r}} q_{\mathrm{i} 2}^{3}-t^{2} y_{\mathrm{r}} q_{\mathrm{i} 2}^{3}\right.$ $-t_{\mathrm{r}}^{2} y q_{\mathrm{i} 1} q_{\mathrm{i} 2}^{2}-t t_{\mathrm{i}} y_{\mathrm{i}} q_{\mathrm{i} 1} q_{\mathrm{i} 2}^{2}+t^{2} y_{\mathrm{i}} q_{\mathrm{r} 2} q_{\mathrm{i} 2}^{2}+t t_{\mathrm{r}} y q_{\mathrm{i} 1}^{2} q_{\mathrm{i} 2}+t t_{\mathrm{r}} y q_{\mathrm{r} 1}^{2} q_{\mathrm{i} 2}-t_{\mathrm{i}} t_{\mathrm{r}} y_{\mathrm{i}} q_{\mathrm{r} 1}^{2} q_{\mathrm{i} 2}-t^{2} y_{\mathrm{r}} q_{\mathrm{r} 2}^{2} q_{\mathrm{i} 2}+t^{2} y_{\mathrm{i}} q_{\mathrm{r} 2}^{3}-t_{\mathrm{r}}^{2} y_{\mathrm{i}} q_{\mathrm{r} 2}^{3}$ $\left.+t_{\mathrm{i}}^{2} y q_{\mathrm{i} 1} q_{\mathrm{r} 2}^{2}+t t_{\mathrm{r}} y_{\mathrm{r}} q_{\mathrm{i} 1} q_{\mathrm{r} 2}^{2}-t t_{\mathrm{i}} y q_{\mathrm{i} 1}^{2} q_{\mathrm{r} 2}-t t_{\mathrm{i}} y q_{\mathrm{r} 1}^{2} q_{\mathrm{r} 2}+t_{\mathrm{i}} t_{\mathrm{r}} y_{\mathrm{r}} q_{\mathrm{r} 1}^{2} q_{\mathrm{r} 2}\right)+6\left(-y_{\mathrm{i}} q_{\mathrm{i} 1} q_{\mathrm{i} 2} q_{\mathrm{r} 1} t_{\mathrm{i}}^{2}+y_{\mathrm{i}} q_{\mathrm{i} 1}^{2} q_{\mathrm{r} 2} t_{\mathrm{i}}^{2}+y_{\mathrm{r}} q_{3} q_{\mathrm{i} 1} q_{\mathrm{r} 2} t_{\mathrm{i}}^{2}\right.$ $\left.+y_{\mathrm{r}} q_{\mathrm{i} 1} q_{\mathrm{r} 1} q_{\mathrm{r} 2} t_{\mathrm{i}}^{2}-t_{\mathrm{r}}^{2} y_{\mathrm{r}} q_{\mathrm{i} 1}^{2} q_{\mathrm{i} 2}-t_{\mathrm{r}}^{2} y_{\mathrm{i}} q_{3} q_{\mathrm{i} 1} q_{\mathrm{i} 2}+t_{\mathrm{r}}^{2} y_{\mathrm{i}} q_{\mathrm{i} 1} q_{\mathrm{i} 2} q_{\mathrm{r} 1}-t_{\mathrm{r}}^{2} y_{\mathrm{r}} q_{\mathrm{i} 1} q_{\mathrm{r} 1} q_{\mathrm{r} 2}\right)-4\left(-t_{\mathrm{i}} t_{\mathrm{r}} y_{\mathrm{i}} q_{\mathrm{i} 2} q_{\mathrm{i} 1}^{2}-t^{2} y_{\mathrm{r}} q_{\mathrm{i} 2} q_{\mathrm{i} 1}^{2}\right.$ $+t^{2} y_{\mathrm{i}} q_{\mathrm{r} 2} q_{\mathrm{i} 1}^{2}+t_{\mathrm{i}} t_{\mathrm{r}} y_{\mathrm{r}} q_{\mathrm{r} 2} q_{\mathrm{i} 1}^{2}+t_{\mathrm{i}}^{2} y q_{\mathrm{i} 2}^{2} q_{\mathrm{i} 1}-t_{\mathrm{r}}^{2} y q_{\mathrm{r} 2}^{2} q_{\mathrm{i} 1}-t^{2} y_{\mathrm{r}} q_{\mathrm{i} 2} q_{\mathrm{r} 1}^{2}+t_{\mathrm{i}}^{2} y_{\mathrm{r}} q_{\mathrm{i} 2} q_{\mathrm{r} 1}^{2}-t_{\mathrm{i}} t_{\mathrm{r}} y q_{\mathrm{r} 1} q_{\mathrm{r} 2}^{2}-t t_{\mathrm{r}} y_{\mathrm{i}} q_{\mathrm{r} 1} q_{\mathrm{r} 2}^{2}$ $\left.-t t_{\mathrm{i}} y_{\mathrm{r}} q_{\mathrm{r} 1} q_{\mathrm{r} 2}^{2}-t_{\mathrm{i}} t_{\mathrm{r}} y q_{\mathrm{i} 2}^{2} q_{\mathrm{r} 1}-t t_{\mathrm{r}} y_{\mathrm{i}} q_{\mathrm{i} 2}^{2} q_{\mathrm{r} 1}-t t_{\mathrm{i}} y_{\mathrm{r}} q_{\mathrm{i} 2}^{2} q_{\mathrm{r} 1}+t^{2} y_{\mathrm{i}} q_{\mathrm{r} 1}^{2} q_{\mathrm{r} 2}-t_{\mathrm{r}}^{2} y_{\mathrm{i}} q_{\mathrm{r} 1}^{2} q_{\mathrm{r} 2}\right)-2\left(t t_{\mathrm{i}} y_{\mathrm{i}} q_{\mathrm{i} 1}^{3}-t t_{\mathrm{r}} y_{\mathrm{r}} q_{\mathrm{i} 1}^{3}\right.$ $-t_{\mathrm{i}}^{2} y_{\mathrm{r}} q_{\mathrm{i} 2} q_{\mathrm{i} 1}^{2}+t_{\mathrm{i}} t_{\mathrm{r}} y q_{\mathrm{r} 1} q_{\mathrm{i} 1}^{2}+t t_{\mathrm{r}} y_{\mathrm{i}} q_{\mathrm{r} 1} q_{\mathrm{i} 1}^{2}+t t_{\mathrm{i}} y_{\mathrm{r}} q_{\mathrm{r} 1} q_{\mathrm{i} 1}^{2}+t_{\mathrm{r}}^{2} y_{\mathrm{i}} q_{\mathrm{r} 2} q_{\mathrm{i} 1}^{2}+t t_{\mathrm{i}} y_{\mathrm{i}} q_{\mathrm{r} 1}^{2} q_{\mathrm{i} 1}-t t_{\mathrm{r}} y_{\mathrm{r}} q_{\mathrm{r} 1}^{2} q_{\mathrm{i} 1}+t_{\mathrm{i}} t_{\mathrm{r}} y q_{\mathrm{r} 1}^{3}+t t_{\mathrm{r}} y_{\mathrm{i}} q_{\mathrm{r} 1}^{3}$ $\left.\left.+t t_{\mathrm{i}} y_{\mathrm{r}} q_{\mathrm{r} 1}^{3}\right)-t_{\mathrm{i}}^{2} y q_{\mathrm{i} 1}^{3}+t_{\mathrm{r}}^{2} y q_{\mathrm{i} 1}^{3}-t_{\mathrm{i}}^{2} y q_{\mathrm{r} 1}^{2} q_{\mathrm{i} 1}+t_{\mathrm{r}}^{2} y q_{\mathrm{r} 1}^{2} q_{\mathrm{i} 1}\right]$ 


\section{E Syzygies}

Here we list syzygies that we have explicitly constructed in the course of this work.

\begin{tabular}{|c|c|c|c|c|c|c|c|c|}
\hline order & structure & $\mathrm{CP}$ & HS & $\mathrm{PL}$ & $\mathrm{PP}$ & new rels. & old rels. & comments \\
\hline 6 & $q^{2} y^{2} t^{2}$ & + & 6 & -1 & 7 & 1 & - & $\mathcal{I}_{1,1,1}^{2}$, eq. $(6.2)$ \\
\hline \multirow[t]{2}{*}{7} & $q^{3} y^{2} t^{2}$ & + & 7 & -1 & 8 & 1 & - & \\
\hline & $q^{2} y^{3} t^{2} / q^{2} y^{2} t^{3}$ & - & 3 & -1 & 4 & $1 / 1$ & - & eq. (7.2) \\
\hline \multirow[t]{5}{*}{8} & $q^{2} y^{4} t^{2} / q^{2} y^{2} t^{4}$ & + & 9 & -1 & 11 & $1 / 1$ & $1 / 1$ & $\mathcal{J}_{1,2,1}^{2} / \mathcal{J}_{1,1,2}^{2}$ \\
\hline & $q^{2} y^{3} t^{3}$ & + & 9 & -1 & 10 & 1 & - & $\mathcal{J}_{1,2,1} \times \mathcal{J}_{1,1,2}$ \\
\hline & $q^{4} y^{2} t^{2}$ & + & 10 & -1 & 12 & 1 & 1 & $\mathcal{I}_{2,1,1}^{2}-\left(\mathcal{I}_{2,2,0} \times \mathcal{I}_{2,0,2}\right)$ \\
\hline & $q^{3} y^{4} t / q^{3} y t^{4}$ & - & 4 & -1 & 5 & $1 / 1$ & - & $\mathcal{I}_{2,2,0} \mathcal{J}_{1,2,1} / \mathcal{I}_{2,0,2} \mathcal{J}_{1,1,2}$, eq. $(7.3)$ \\
\hline & $q^{3} y^{3} t^{2} / q^{3} y^{2} t^{3}$ & - & 6 & -3 & 9 & $3 / 3$ & - & $\begin{array}{l}\mathcal{I}_{2,2,0} \mathcal{J}_{1,1,2} / \mathcal{I}_{2,0,2} \mathcal{J}_{1,2,1} \\
\mathcal{I}_{2,1,1} \mathcal{J}_{1,2,1} / \mathcal{I}_{2,1,1} \mathcal{J}_{1,1,2}\end{array}$ \\
\hline \multirow[t]{4}{*}{9} & $q^{3} y^{3} t^{3}$ & + & 13 & -2 & 17 & 2 & 2 & \\
\hline & $q^{3} y^{4} t^{2} / q^{3} y^{2} t^{4}$ & + & 12 & -1 & 15 & $1 / 1$ & $2 / 2$ & \\
\hline & $q^{4} y^{4} t / q^{4} y t^{4}$ & - & 5 & -1 & 6 & $1 / 1$ & - & \\
\hline & $q^{4} y^{3} t^{2} / q^{4} y^{2} t^{3}$ & - & 7 & -3 & 11 & $3 / 3$ & $1 / 1$ & \\
\hline \multirow[t]{4}{*}{10} & $q^{4} y^{4} t^{2} / q^{4} y^{2} t^{4}$ & + & 18 & -3 & 22 & $3 / 3$ & $1 / 1$ & $\mathcal{J}_{2,2,1}^{2} / \mathcal{J}_{2,1,2}^{2}$, eq. $(7.4)$ \\
\hline & $q^{5} y t^{4} / q^{5} y^{4} t$ & - & 7 & -1 & 9 & $1 / 1$ & $1 / 1$ & \\
\hline & $q^{5} y^{3} t^{2} / q^{5} y^{2} t^{3}$ & - & 10 & -2 & 13 & $2 / 2$ & $1 / 1$ & \\
\hline & $\vdots$ & & & $\vdots$ & & $\vdots$ & $\vdots$ & \\
\hline \multirow[t]{4}{*}{12} & $q^{6} y^{6} / q^{6} t^{6}$ & + & 10 & -1 & 11 & $1 / 1$ & - & $\mathcal{J}_{3,3,0}^{2} / \mathcal{J}_{3,0,3}^{2}$ \\
\hline & $q^{6} y^{4} t^{2} / q^{6} y^{2} t^{4}$ & + & 27 & -2 & 38 & $2 / 2$ & $9 / 9$ & $\mathcal{J}_{3,2,1}^{2} / \mathcal{J}_{3,1,2}^{2}$ \\
\hline & $q^{6} y^{3} t^{3}$ & + & 28 & -2 & 43 & 2 & 13 & $\mathcal{J}_{3,3,0} \times \mathcal{J}_{3,0,3}, \mathcal{J}_{3,2,1} \times \mathcal{J}_{3,1,2}$ \\
\hline & & & & : & & $\vdots$ & $\vdots$ & \\
\hline
\end{tabular}

Table 1. List of the syzygies of the 2HDM scalar sector. The first columns give the order, structure and $\mathrm{CP}$ transformation behavior of the relation. The following columns summarize the corresponding coefficients of the multi-graded Hilbert series (HS), Plethystic logarithm (PL) as well as the number of new relations that are arising (as evidence that the PL coefficient indeed gives this number correctly). Furthermore, we list the total number of power products of generating invariants which give rise to the same structure (this is an indicator of how many previously found "old" relations appear in addition to possible new relations). The last column gives important structures of invariants that appear in the given syzygy.

Open Access. This article is distributed under the terms of the Creative Commons Attribution License (CC-BY 4.0), which permits any use, distribution and reproduction in any medium, provided the original author(s) and source are credited. 


\section{References}

[1] G.C. Branco, P.M. Ferreira, L. Lavoura, M.N. Rebelo, M. Sher and J.P. Silva, Theory and phenomenology of two-Higgs-doublet models, Phys. Rept. 516 (2012) 1 [arXiv:1106.0034] [INSPIRE].

[2] A. Santamaria, Masses, mixings, Yukawa couplings and their symmetries, Phys. Lett. B 305 (1993) 90 [hep-ph/9302301] [INSPIRE].

[3] G.C. Branco, L. Lavoura and J.P. Silva, CP Violation, Int. Ser. Monogr. Phys. 103 (1999) 1 [INSPIRE].

[4] C. Jarlskog, Commutator of the Quark Mass Matrices in the Standard Electroweak Model and a Measure of Maximal CP-violation, Phys. Rev. Lett. 55 (1985) 1039 [InSPIRE].

[5] J. Bernabeu, G.C. Branco and M. Gronau, CP Restrictions on Quark Mass Matrices, Phys. Lett. 169B (1986) 243 [INSPIRE].

[6] G.C. Branco, L. Lavoura and M.N. Rebelo, Majorana Neutrinos and CP Violation in the Leptonic Sector, Phys. Lett. B 180 (1986) 264 [INSPIRE].

[7] F.J. Botella and J.P. Silva, Jarlskog-like invariants for theories with scalars and fermions, Phys. Rev. D 51 (1995) 3870 [hep-ph/9411288] [INSPIRE].

[8] L. Lavoura and J.P. Silva, Fundamental CP-violating quantities in a $\mathrm{SU}(2) \times \mathrm{U}(1)$ model with many Higgs doublets, Phys. Rev. D 50 (1994) 4619 [hep-ph/9404276] [INSPIRE].

[9] J.F. Gunion and H.E. Haber, Conditions for CP-violation in the general two-Higgs-doublet model, Phys. Rev. D 72 (2005) 095002 [hep-ph/0506227] [INSPIRE].

[10] G.C. Branco, M.N. Rebelo and J.I. Silva-Marcos, CP-odd invariants in models with several Higgs doublets, Phys. Lett. B 614 (2005) 187 [hep-ph/0502118] [INSPIRE].

[11] I.P. Ivanov, Two-Higgs-doublet model from the group-theoretic perspective, Phys. Lett. $\mathbf{B} 632$ (2006) 360 [hep-ph/0507132] [INSPIRE].

[12] C.C. Nishi, CP violation conditions in N-Higgs-doublet potentials, Phys. Rev. D 74 (2006) 036003 [Erratum ibid. D 76 (2007) 119901] [hep-ph/0605153] [INSPIRE].

[13] O. Lebedev, CP violating invariants in supersymmetry, Phys. Rev. D 67 (2003) 015013 [hep-ph/0209023] [INSPIRE].

[14] H.K. Dreiner, J.S. Kim, O. Lebedev and M. Thormeier, Supersymmetric Jarlskog invariants: The Neutrino sector, Phys. Rev. D 76 (2007) 015006 [hep-ph/0703074] [INSPIRE].

[15] I.P. Ivanov and J.P. Silva, CP-conserving multi-Higgs model with irremovable complex coefficients, Phys. Rev. D 93 (2016) 095014 [arXiv:1512.09276] [INSPIRE].

[16] H.E. Haber, O.M. Ogreid, P. Osland and M.N. Rebelo, Symmetries and Mass Degeneracies in the Scalar Sector, JHEP 01 (2019) 042 [arXiv: 1808.08629] [INSPIRE].

[17] I.P. Ivanov, C.C. Nishi, J.P. Silva and A. Trautner, Basis-invariant conditions for CP symmetry of order four, Phys. Rev. D 99 (2019) 015039 [arXiv:1810.13396] [InSPIRE].

[18] E.E. Jenkins and A.V. Manohar, Rephasing Invariants of Quark and Lepton Mixing Matrices, Nucl. Phys. B 792 (2008) 187 [arXiv:0706.4313] [InSPIRE].

[19] E.E. Jenkins and A.V. Manohar, Algebraic Structure of Lepton and Quark Flavor Invariants and CP-violation, JHEP 10 (2009) 094 [arXiv:0907.4763] [INSPIRE]. 
[20] A. Hanany, E.E. Jenkins, A.V. Manohar and G. Torri, Hilbert Series for Flavor Invariants of the Standard Model, JHEP 03 (2011) 096 [arXiv: 1010.3161] [INSPIRE].

[21] S. Davidson and H.E. Haber, Basis-independent methods for the two-Higgs-doublet model, Phys. Rev. D 72 (2005) 035004 [Erratum ibid. D 72 (2005) 099902] [hep-ph/0504050] [INSPIRE].

[22] H.E. Haber and D. O'Neil, Basis-independent methods for the two-Higgs-doublet model. II. The Significance of $\tan \beta$, Phys. Rev. D 74 (2006) 015018 [Erratum ibid. D 74 (2006) 059905] [hep-ph/0602242] [INSPIRE].

[23] B. Grzadkowski, O.M. Ogreid and P. Osland, Spontaneous CP-violation in the 2HDM: physical conditions and the alignment limit, Phys. Rev. D 94 (2016) 115002 [arXiv: 1609.04764] [INSPIRE].

[24] M.P. Bento, H.E. Haber, J.C. Romão and J.P. Silva, Multi-Higgs doublet models: physical parametrization, sum rules and unitarity bounds, JHEP 11 (2017) 095 [arXiv:1708.09408] [INSPIRE].

[25] O.M. Ogreid, Invariants and CP-violation in the 2HDM, PoS (CORFU2017) 065 [arXiv: 1803.09351] [INSPIRE].

[26] T. Feldmann, T. Mannel and S. Schwertfeger, Renormalization Group Evolution of Flavour Invariants, JHEP 10 (2015) 007 [arXiv: 1507.00328] [INSPIRE].

[27] S.H. Chiu and T.K. Kuo, Renormalization of the Neutrino Mass Matrix, Phys. Lett. B 760 (2016) 544 [arXiv: 1510.07368] [INSPIRE].

[28] S.H. Chiu and T.K. Kuo, Renormalization of the quark mass matrix, Phys. Rev. D 93 (2016) 093006 [arXiv: 1603.04568] [INSPIRE].

[29] F. Herren, L. Mihaila and M. Steinhauser, Gauge and Yukawa coupling $\beta$-functions of two-Higgs-doublet models to three-loop order, Phys. Rev. D 97 (2018) 015016 [arXiv: 1712.06614] [INSPIRE].

[30] A.V. Bednyakov, On three-loop RGE for the Higgs sector of 2HDM, arXiv:1809.04527 [INSPIRE].

[31] J. Bijnens, J. Oredsson and J. Rathsman, Scalar Kinetic Mixing and the Renormalization Group, Phys. Lett. B 792 (2019) 238 [arXiv:1810.04483] [INSPIRE].

[32] I. de Medeiros Varzielas, S.F. King, C. Luhn and T. Neder, CP-odd invariants for multi-Higgs models: applications with discrete symmetry, Phys. Rev. D 94 (2016) 056007 [arXiv: 1603.06942] [INSPIRE].

[33] D. Berger, J.N. Howard and A. Rajaraman, Invariant Tensors in Gauge Theories, LHEP 1 (2018) 14 [arXiv:1806.04332] [INSPIRE].

[34] R.P. Stanley, Invariants of finite groups and their applications to combinatorics, Bull. Am. Math. Soc. (N.S.) 1 (1979) 475.

[35] B. Sturmfels, Algorithms in invariant theory. Texts and Monographs in Symbolic Computation, second edition, Springer, Berlin Germany (2008).

[36] E. Getzler and M.M. Kapranov, Modular operads, dg-ga/9408003 [INSPIRE].

[37] J.M.F. Labastida and M. Marino, A New Point of View in the Theory of Knot and Link Invariants, math/0104180 [INSPIRE]. 
[38] S. Benvenuti, B. Feng, A. Hanany and Y.-H. He, Counting BPS Operators in Gauge Theories: Quivers, Syzygies and Plethystics, JHEP 11 (2007) 050 [hep-th/0608050] [INSPIRE].

[39] B. Feng, A. Hanany and Y.-H. He, Counting gauge invariants: The Plethystic program, JHEP 03 (2007) 090 [hep-th/0701063] [INSPIRE].

[40] Y. Noma, T. Nakatsu and T. Tamakoshi, Plethystics and instantons on ALE spaces, hep-th/0611324 [INSPIRE].

[41] A. Butti, D. Forcella, A. Hanany, D. Vegh and A. Zaffaroni, Counting Chiral Operators in Quiver Gauge Theories, JHEP 11 (2007) 092 [arXiv:0705.2771] [INSPIRE].

[42] J. Gray, A. Hanany, Y.-H. He, V. Jejjala and N. Mekareeya, SQCD: A Geometric Apercu, JHEP 05 (2008) 099 [arXiv: 0803.4257] [INSPIRE].

[43] A. Hanany and N. Mekareeya, Counting Gauge Invariant Operators in SQCD with Classical Gauge Groups, JHEP 10 (2008) 012 [arXiv:0805.3728] [INSPIRE].

[44] A. Hanany, N. Mekareeya and G. Torri, The Hilbert Series of Adjoint SQCD, Nucl. Phys. B 825 (2010) 52 [arXiv:0812.2315] [INSPIRE].

[45] A. Hanany and R. Kalveks, Highest Weight Generating Functions for Hilbert Series, JHEP 10 (2014) 152 [arXiv: 1408.4690] [INSPIRE].

[46] A. Bourget and A. Pini, Non-Connected Gauge Groups and the Plethystic Program, JHEP 10 (2017) 033 [arXiv:1706.03781] [INSPIRE].

[47] B. Henning, X. Lu, T. Melia and H. Murayama, Hilbert series and operator bases with derivatives in effective field theories, Commun. Math. Phys. 347 (2016) 363 [arXiv: 1507.07240] [INSPIRE].

[48] B. Henning, X. Lu, T. Melia and H. Murayama, 2, 84, 30, 993, 560, 15456, 11962, 261485, ...: Higher dimension operators in the SM EFT, JHEP 08 (2017) 016 [arXiv:1512.03433] [INSPIRE].

[49] B. Henning, X. Lu, T. Melia and H. Murayama, Operator bases, S-matrices and their partition functions, JHEP 10 (2017) 199 [arXiv:1706.08520] [INSPIRE].

[50] L. Lehman and A. Martin, Hilbert Series for Constructing Lagrangians: expanding the phenomenologist's toolbox, Phys. Rev. D 91 (2015) 105014 [arXiv: 1503.07537] [INSPIRE].

[51] R.M. Fonseca, Calculating the renormalisation group equations of a SUSY model with Susyno, Comput. Phys. Commun. 183 (2012) 2298 [arXiv:1106.5016] [INSPIRE].

[52] S. Keppeler and M. Sjödahl, Hermitian Young Operators, J. Math. Phys. 55 (2014) 021702 [arXiv: 1307.6147] [INSPIRE].

[53] J. Alcock-Zeilinger and H. Weigert, Simplification Rules for Birdtrack Operators, J. Math. Phys. 58 (2017) 051701 [arXiv:1610.08801] [INSPIRE].

[54] J. Alcock-Zeilinger and H. Weigert, Transition Operators, J. Math. Phys. 58 (2017) 051703 [arXiv: 1610.08802] [INSPIRE].

[55] J. Alcock-Zeilinger and H. Weigert, Compact Hermitian Young Projection Operators, J. Math. Phys. 58 (2017) 051702 [arXiv: 1610.10088] [INSPIRE].

[56] P. Cvitanovic, Group theory for Feynman diagrams in non-Abelian gauge theories, Phys. Rev. D 14 (1976) 1536 [INSPIRE]. 
[57] P. Cvitanovic, Group theory: Birdtracks, Lie's and exceptional groups, Princeton University Press, Princeton U.S.A. (2008).

[58] S. Keppeler, Birdtracks for SU(N), in QCD Master Class 2017, Saint-Jacut-de-la-Mer France (2017) [arXiv: 1707.07280] [INSPIRE].

[59] E. Noether, Der Endlichkeitssatz der Invarianten endlicher Gruppen, Math. Ann. 77 (1916) 89.

[60] M. Hochster and J.L. Roberts, Actions of reductive groups on regular rings and Cohen-Macaulay rings, Bull. Am. Math. Soc. 80 (1974) 281.

[61] M. Hochster and J.L. Roberts, Rings of invariants of reductive groups acting on regular rings are Cohen-Macaulay, Adv. Math. 13 (1974) 115.

[62] F. Knop and P. Littelmann, Der Grad erzeugender Funktionen von Invariantenringen, Math. Z. 196 (1987) 211.

[63] F. Knop, Der kanonische modul eines invariantenrings, J. Algebra 127 (1989) 40.

[64] H.K. Dreiner, H.E. Haber and S.P. Martin, Two-component spinor techniques and Feynman rules for quantum field theory and supersymmetry, Phys. Rept. 494 (2010) 1 [arXiv:0812.1594] [INSPIRE].

[65] G. Ecker, W. Grimus and H. Neufeld, A Standard Form for Generalized CP Transformations, J. Phys. A 20 (1987) L807 [InSPIRE].

[66] A. Bourget, A. Pini and D. Rodríguez-Gómez, Gauge theories from principally extended disconnected gauge groups, Nucl. Phys. B 940 (2019) 351 [arXiv:1804.01108] [INSPIRE].

[67] N.M. Thiéry, Algebraic invariants of graphs; a study based on computer exploration, arXiv:0812.3082.

[68] S.A. King, Fast Computation of Secondary Invariants, math/0701270.

[69] F. Nagel, New aspects of gauge-boson couplings and the Higgs sector, Ph.D. Thesis, Heidelberg University, Heidelberg Germany (2004).

[70] M. Maniatis, A. von Manteuffel, O. Nachtmann and F. Nagel, Stability and symmetry breaking in the general two-Higgs-doublet model, Eur. Phys. J. C 48 (2006) 805 [hep-ph/0605184] [INSPIRE].

[71] I.P. Ivanov, Minkowski space structure of the Higgs potential in 2HDM, Phys. Rev. D 75 (2007) 035001 [Erratum ibid. D 76 (2007) 039902] [hep-ph/0609018] [INSPIRE].

[72] M. Maniatis, A. von Manteuffel and O. Nachtmann, CP violation in the general two-Higgs-doublet model: A Geometric view, Eur. Phys. J. C 57 (2008) 719 [arXiv: 0707.3344] [INSPIRE].

[73] I.P. Ivanov, Minkowski space structure of the Higgs potential in 2HDM. II. Minima, symmetries and topology, Phys. Rev. D 77 (2008) 015017 [arXiv:0710.3490] [INSPIRE].

[74] P.M. Ferreira, M. Maniatis, O. Nachtmann and J.P. Silva, CP properties of symmetry-constrained two-Higgs-doublet models, JHEP 08 (2010) 125 [arXiv:1004.3207] [INSPIRE].

[75] P.M. Ferreira, H.E. Haber, M. Maniatis, O. Nachtmann and J.P. Silva, Geometric picture of generalized-CP and Higgs-family transformations in the two-Higgs-doublet model, Int. J. Mod. Phys. A 26 (2011) 769 [arXiv:1010.0935] [inSPIRE]. 
[76] M. Maniatis and O. Nachtmann, Stability and symmetry breaking in the general three-Higgs-doublet model, JHEP 02 (2015) 058 [Erratum ibid. 1510 (2015) 149] [arXiv: 1408.6833] [INSPIRE].

[77] I.P. Ivanov and C.C. Nishi, Symmetry breaking patterns in 3HDM, JHEP 01 (2015) 021 [arXiv: 1410.6139] [INSPIRE].

[78] R. Ehrenborg and G.-C. Rota, Apolarity and Canonical Forms for Homogeneous Polynomials, Eur. J. Comb. 14 (1993) 157.

[79] M. Beecken, J. Mittmann, and N. Saxena, Algebraic Independence and Blackbox Identity Testing, arXiv:1102.2789. 